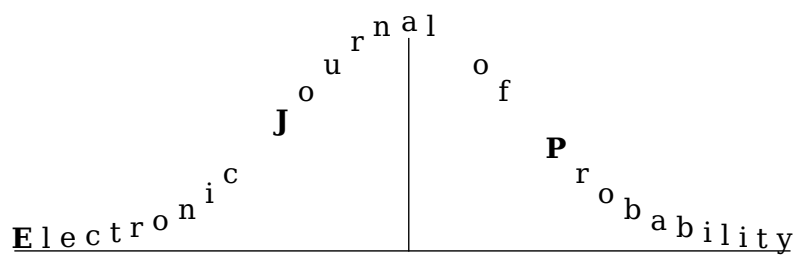

Electron. J. Probab. 25 (2020), article no. 76, 1-35.

ISSN: 1083-6489 https://doi.org/10.1214/20-EJP473

\title{
Hydrodynamic limit of a $(2+1)$-dimensional crystal growth model in the anisotropic KPZ class*
}

\author{
Vincent Lerouvillois ${ }^{\dagger}$
}

\begin{abstract}
We study a model, introduced initially by Gates and Westcott [11] to describe crystal growth evolution, which belongs to the Anisotropic KPZ universality class [19]. It can be thought of as a $(2+1)$-dimensional generalisation of the well known $(1+1)$ dimensional Polynuclear Growth Model (PNG). We show the full hydrodynamic limit of this process i.e the convergence of the random interface height profile after ballistic space-time scaling to the viscosity solution of a Hamilton-Jacobi PDE: $\partial_{t} u=v(\nabla u)$ with $v$ an explicit non-convex speed function. The convergence holds in the strong almost sure sense.
\end{abstract}

Keywords: interface growth; hydrodynamic limit; anisotropic KPZ. AMS MSC 2010: 60J25; 60K35; 82C24.

Submitted to EJP on October 14, 2019, final version accepted on May 27, 2020.

Supersedes arXiv: 1910.01015.

Supersedes HAL : hal -02307320.

\section{Introduction}

Crystal growth belongs to a wider class of random interface growth phenomena that appear naturally in physics and biology [1]. Trying to better understand the behavior of these natural phenomena is a source of interest in itself. On the other hand, random growth models mainly caught the attention of mathematicians in the last couple of decades because of their conjectural universality properties and relation with the KPZ (Kardar-Parisi-Zhang) equation [14] which presumably encodes their long-time fluctuation behavior (see e.g. [5, 10, 21] for reviews on the topic in dimension $(1+1)$ and [28] in dimension $(2+1)$ ).

To fix ideas, the microscopic $d$-dimensional interface is typically modelled by the graph of a discrete height function $h: \mathbb{Z}^{d} \times \mathbb{R}_{+} \rightarrow \mathbb{Z}$ (here, $\mathbb{R}_{+}$represents the time variable) and evolves according to an asymmetric Markovian dynamic which is often

\footnotetext{
*Partially supported by ANR-15-CE40-0020-03 Grant LSD.

${ }^{\dagger}$ Univ Lyon, Université Claude Bernard Lyon 1, CNRS UMR 5208, Institut Camille Jordan, F-69622 Villeurbanne, France. E-mail: lerouvillois@math.univ-lyon1.fr
} 
related to interacting particles systems. The transition rates are assumed to depend only on height gradients, so that the dynamics is invariant by vertical translations of the interface. The first problem one may address is the law of large numbers or hydrodynamic limit, i.e the typical macroscopic behavior of the randomly evolving height function. Under space-time ballistic rescaling of the form $n^{-1} h(\lfloor n x\rfloor, n t)$, the height profile is expected to converge to the solution of a first-order non-linear PDE of Hamilton-Jacobi type:

$$
\partial_{t} u=v(\nabla u),
$$

where the growth velocity $v$ only depends on the slope and not on $u$ itself since the model is vertically translation invariant. Next, and more challengingly, comes the study of fluctuations, i.e the behavior of the discrete height function around its hydrodynamic limit. The large-scale fluctuations are expected to look qualitatively like the solution of the KPZ equation and in particular share the same universal characteristics exponents. Most results in this direction are established for $d=1$. In dimension two, growth models are conjectured [29] to fall into two universality classes depending on the convexity properties of $v$. When $v$ is strictly convex (or concave), we speak simply of KPZ universality class: it is predicted and numerically observed that fluctuations grow like $t^{\beta}$ with a universal exponent $\beta>0$ and spatial fluctuations at equilibrium grow with a "roughness exponent" $\alpha=2 \beta /(\beta+1)$. When the Hessian of $v$ has signature $(+,-)$ the model is conjectured to belong to the so-called Anisotropic KPZ (AKPZ) class where spatial and temporal fluctuations are expected to grow logarithmically and spatial fluctuations to scale to a Gaussian Free Field, as is the case for the stochastic heat equation with additive noise. One says that the non-linearity in the KPZ equation is irrelevant in the AKPZ regime and relevant in the $\mathrm{KPZ}$ one.

The model we are considering in this paper was introduced by Gates and Westcott in [11] to describe crystal growth evolution and its stationary states. The interface can be described by a height function $h:(\mathbb{R} \times \mathbb{Z}) \times \mathbb{R}_{+} \rightarrow \mathbb{Z}$, semi-discrete in space and continuous in time, whose level lines are piece-wise constant functions with \pm 1 jumps. Even if we adopt a different viewpoint, the Gates-Westcott dynamic can be viewed as a multi-line generalisation of the PNG dynamic where each level line follows simultaneously the PNG dynamic with "kink/antikink creations" suppressed whenever two lines intersect. Although the PNG is a solvable model that can be mapped to the problem of the longest increasing subsequence of a random permutation, to random polymers and to random matrix ensembles (see [9] for a nice review on the topic), the Gates-Westcott dynamics induces non-trivial interaction among level lines, which makes the model harder to analyse. In [19], Prähofer and Spohn identified a slope-dependent family of stationary distributions for the dynamic restricted to a bi-dimensional torus (note that Gates and Westcott already computed equilibrium measures in [11] but only for a one-dimensional subset of slopes $\rho$ ). In a certain thermodynamic limit of large torus, they were able to compute the slope-dependent growth velocity $v(\rho)$ at stationarity. This is the natural candidate for the speed function $v(\rho)$ in (1.1). As expected, the Hessian of $v$ has signature $(+,-)$ everywhere so the model belongs to the AKPZ universality class. The authors of [19] also showed that the spatial fluctuations at equilibrium are of logarithmic order with respect to the distance between points; this is typical of the two-dimensional Gaussian Free Field. However, they didn't treat the temporal fluctuations (also expected to grow logarithmically). Our contribution to the study of the model is the rigorous proof of the hydrodynamic limit starting from arbitrary initial condition. As an intermediate step, we also get a logarithmic upper bound on fluctuation growth w.r.t. time in the stationary states (see Lemma 6.4). 
In the literature, most results about hydrodynamic limits in multi-dimensional spaces are given for convex velocities $v$, where the viscosity solution of (1.1) can be expressed in terms of the variational Hopf-Lax formula. The strategy is to show that the discrete height function enjoys a variational formula (sometimes called "envelope property") at the microscopic level, which passes to the limit thanks to the sub-additive ergodic theorem. This applies e.g. to the Corner Growth Model [26, Section 9], Ballistic deposition [25] and a wider family of grows models on $\mathbb{Z}^{d}$ [23], and yields existence of such a hydrodynamic limit without providing an explicit expression of the speed function $v$. The function $v$ can be explicitly identified when equilibrium measures are known, as is the case for various one-dimensional models, such as ASEP and PNG. For two-dimensional models in the AKPZ class, such envelope property and Hopf-Lax formula cannot hold, otherwise the speed function in the hydrodynamic limit would automatically result to be convex.

In his seminal article [22], Rezakhanlou introduced a different approach to hydrodynamic limit for growth processes based on a compactness argument and on a list of conditions that allow to identify any limit point with the unique viscosity solution of (1.1). This method does not require convexity of $v$, but the only examples for which a full hydrodynamic limit was proved in [22] are one-dimensional where the structure of ergodic translation invariant stationary measures is better understood. For $d \geq 2$, only a partial result was obtained, namely, that any limit in distribution of the rescaled height profile is concentrated on a set of viscosity solutions of Hamilton-Jacobi equations with a possibly random speed function. However, a precise description of equilibrum measures is available for some of these models (e.g. the Gates-Westcott model [19] and models related to the two-dimensional dimer model $[2,3,6,27,4]$ where the stationary measures are given by translation invariant Gibbs measures on perfect matchings [16]). Inspired by Rezakhanlou's technique, Zhang obtained the first full hydrodynamic limit [30] for a $(2+1)$-dimensional growth model. Specifically, he considered the dimer shuffling-algorithm, whose stationary distributions are given by weighted random dimer configurations on $\mathbb{Z}^{2}$. Let us also mention the works [3,17] about a long jump twodimensional interlaced particle dynamic generalising the Hammersley process. In [3], the authors showed the hydrodynamic limit starting from a very specific initial condition (with a CLT for temporal fluctuations on scale $\log t$ ) while in [17], the authors proved the hydrodynamic limit either up to the first time when a shock appears, or under the assumption of a convex initial profile [8].

The present article follows the main idea of $[22,30]$ in terms of proof structure. The idea consists in constructing a sequence (labeled by the parameter $n$ associated to the ballistic rescaling) of discrete random semi-groups associated to the rescaled microscopic dynamic, showing compactness in some sense and identifying the limiting continuous semi-group with the one associated with the unique viscosity solution of the PDE. The identification relies both on the sufficient conditions given in [22] (summarised in Proposition 3.5) and on a precise analysis of the stationary processes. With respect to $[22,30]$, non-trivial additional difficulties we had to overcome in the proof of compactness are related to the semi-continuous character of the model and to unboundedness of the slopes and of the speed function. In particular, we had to control the evolution of spatial gradients (Proposition 5.6) while this was trivial in [22, 30] since gradients are bounded. To do so, we related the height function along the first coordinate to the PNG with a random subset of Poissonian creations and used a representation in terms of random directed polymers. Also, instead of showing tightness of probability measures like in [22, 30], we showed that, for a certain topology, the sequence of random semi-groups is almost surely contained in a (random) compact set and then proved almost sure uniqueness of the possible sub-sequential limits. Let us emphasize that the 
hydrodynamic limit we obtained is in the strong sense of almost sure convergence (on an underlying probability space determined by the Poissonian clocks).

The article is structured as follows. The Gates-Westcott model is introduced in Section 2: we define the state space of admissible height functions and its dynamic via a Poisson Point Process on $\mathbb{R} \times \mathbb{Z} \times \mathbb{R}_{+}$representing space-time locations of kink-antikink creations. In Section 3, we start by stating the main result: the hydrodynamic limit for the height function (Theorem 3.1). Then, we remind elements of Hamilton-Jacobi PDE theory and useful results on equilibrium measures. The rest of the article is dedicated to the proof of the main theorem (the strategy of the proof is briefly explained at the end of Section 3). In Section 4, we first show elementary facts about the microscopic dynamic and a fundamental property of locality (Corollary 4.8) and then construct the sequence of random discrete semi-group mentioned above. Section 5 is about proving compactness. A key step in this proof is the control of random spatio-temporal gradients (Propositions 5.5 and 5.6). Then, we apply a Arzelà-Ascoli type theorem (Proposition D.1) and show compactness of the sequence of discrete semi-groups. Finally, in Section 6, we identify the limit points as the semi-group associated with the unique viscosity solution of (3.5) thanks to Proposition 3.5 and the results about equilibrium measures.

\section{The Gates-Westcott model}

\subsection{Height function}

In this model, the surface will be described by a discrete height function $\varphi: \mathbb{R} \times \mathbb{Z} \rightarrow \mathbb{Z}$ which lives in the state space given as follows:

Definition 2.1. Let $\Gamma$ be the set of functions $h: \mathbb{R} \times \mathbb{Z} \rightarrow \mathbb{Z}$ satisfying the following two conditions:

1. For any $y \in \mathbb{Z}, x \mapsto h(x, y)$ is piece-wise constant with a locally finite number of \pm 1 -valued jumps. By convention, we impose that the values at discontinuity points make the function upper semi-continuous.

2. For any $x \in \mathbb{R}, h(x, y+1)-h(x, y) \in\{-1,0\}$.

Because of condition 1, the discontinuities along direction $x$ can be of three different types:

- kink: $h(x, y)=h\left(x^{-}, y\right)=h\left(x^{+}, y\right)+1$

- antikink: $h(x, y)=h\left(x^{-}, y\right)+1=h\left(x^{+}, y\right)$

- kink-antikink pair: $h(x, y)=h\left(x^{-}, y\right)+1=h\left(x^{+}, y\right)+1$.

A height function looks like a stack of terraces seen from a plane (see Figure 1), the edges of each terrace along the $x$ direction corresponding to the kinks and antikinks of the height function. Due to the first condition in Definition 2.1, each function $h(\cdot, y)$ is entirely determined by the position of its kinks and antikinks and its height at any point $x_{0} \in \mathbb{R}$. In other words, the kinks and antikinks define the variations of the height function along the $x$ direction.

Remark 2.2. In the article [11] of Gates and Westcott, condition 2 was replaced by the height function being integer-valued and non-decreasing along the $y$ direction so that arbitrary slopes could be allowed (which is physically more realistic). However, there exists a one-to-one correspondence between height functions according to these two definition variants, as explained in [20, p. 91]. 


\subsection{Dynamic}

Let $\omega$ be a Poisson Point Process of intensity 2 on $\mathbb{R} \times \mathbb{Z} \times \mathbb{R}_{+}$seen as a random, locally finite, set of points in $\mathbb{R} \times \mathbb{Z} \times \mathbb{R}_{+}$that will be called creations. Starting from a configuration in the state space $\Gamma$, the Gates-Westcott dynamic is defined by three rules: the first two are deterministic while the last one is random.

- Lateral Expansion: each terrace exands laterally at speed 1, i.e. each kink (resp. antikink) of the height function is moved at speed +1 (resp -1 ) along the $x$ direction.

- Annihilation: whenever two terraces meet, they merge, i.e. whenever a kink and an antikink meet, they annihilate each other.

- Creation: If $(x, y, t) \in \omega$, then the height $h$ at $(x, y)$ increases by one at time $t$ if the height function obtained remains in $\Gamma$. In other words, a kink-antikink pair is created at time $t$ and at space position $(x, y)$ if the height function remains in $\Gamma$ after the transition, i.e. if $h\left(x, y-1, t^{-}\right)-h\left(x, y, t^{-}\right)=1, h\left(x, y, t^{-}\right)-h\left(x, y+1, t^{-}\right)=0$ and if there is no preexisting discontinuity of $h\left(\cdot, y, t^{-}\right)$at $x$. Note that the last condition is verified with probability 1 , since the discontinuities are locally finite hence countable for any function in $\Gamma$.

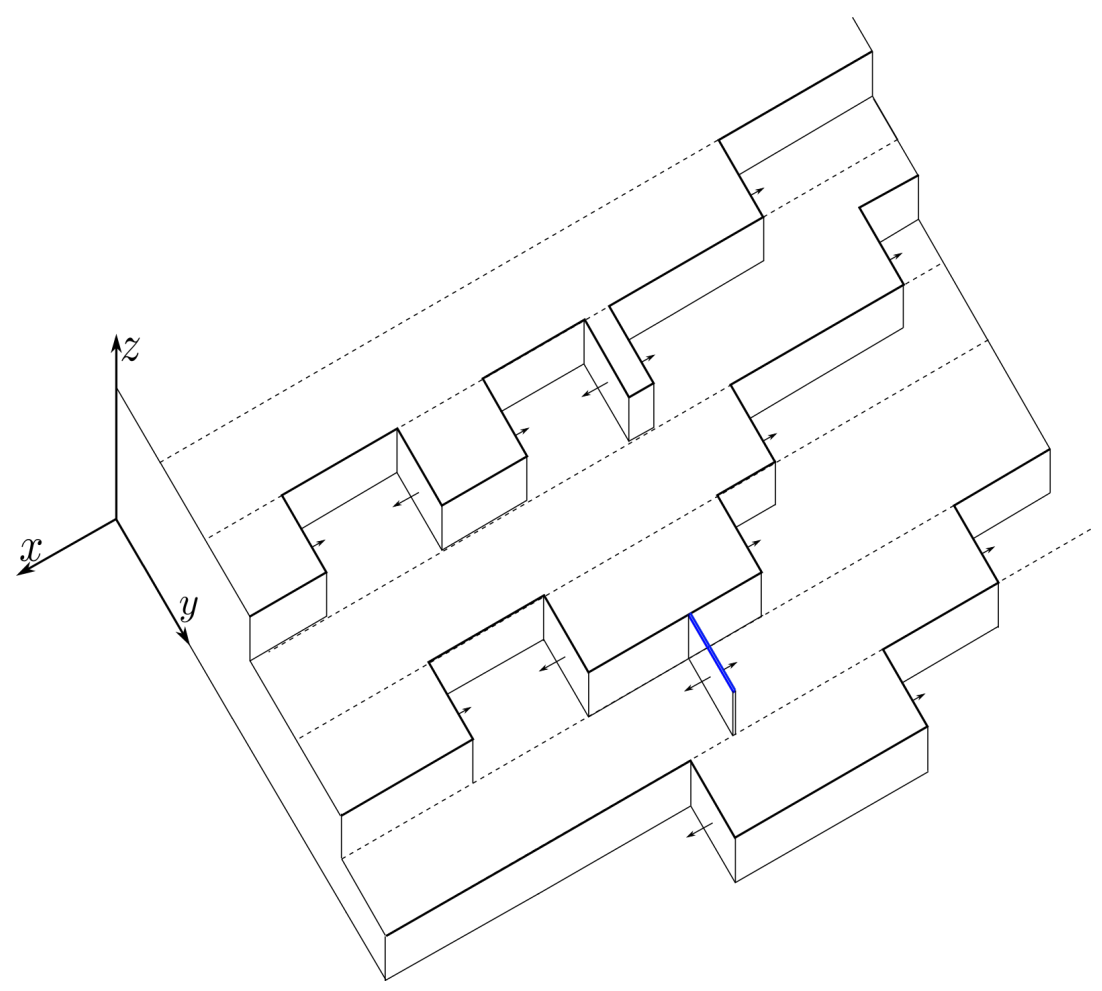

Figure 1: A section of a height function. The lateral expansion is indicated by arrows. A newly created terrace expansion is shown in blue.

Remark 2.3. As usual in interacting particle systems, some care has to be taken to ensure that the process is well defined on the infinite lattice. If we worked in a finite domain, there would be a finite number of creations in finite time intervals and we could know the height function deterministically up to the first time of creation, determine whether this creation occurs or not and repeat the procedure inductively on the number of creations. On the infinite lattice it makes no sense to look at "the first creation" 
but existence and locality of the dynamics can be proven by a modification of the classical disagreement percolation argument [18, Sec. 3.2] used for Glauber dynamics on infinite graphs. Namely, suppose we want to determine the evolution of $h(x, y, t)$ for all $(x, y, t) \in[a, b] \times \llbracket c, d \rrbracket \times[0, T]$. Since kinks/antikinks move with speed 1 , we see that creations whose $x$-coordinate is outside $[a-T, b+T]$ do not matter. Also, let $y^{-}$(resp. $y^{+}$) be the largest integer smaller than $c$ (resp. the smallest integer larger than $d$ ) such that there are no creations at $(x, y, t) \in[a-T, b+T] \times\left\{y^{ \pm}\right\} \times[0, T]$, then the creations that happen for $y>y^{+}$or $y<y^{-}$also do not matter and then the evolution of $h(x, y, t)$ for all $(x, y, t) \in[a, b] \times \llbracket c, d \rrbracket \times[0, T]$ is determined by the finitely many creations in the bounded domain $(x, y, t) \in[a-T, b+T] \times \llbracket y^{-}, y^{+} \rrbracket \times[0, T]$. Finally, for any $a<b \in \mathbb{R}$ and $T>0$, the random variables $y^{ \pm}$are almost surely finite. Later (cf. Proposition 4.5) we will prove a more quantitative locality statement: the height at a point up to time $T$ is determined by creations that occur in a domain that, with high probability, grows linearly with $T$.

Remark 2.4. The Gates-Westcott model can be equivalently described in terms of level lines of the height function (i.e. the bold lines drawn by the terraces edges seen from above in figure 1) as explained in [11, 19, 20]. From this point of view, the dynamic is nothing but the Polynuclear Growth (PNG) Model dynamic [9] applied simultaneously to each level line, creations being suppressed whenever two lines intersect.

\section{The main result}

\subsection{Hydrodynamic limit}

First of all, let us introduce a few definitions and notations. We denote by $\Omega$ the set of locally finite subsets of $\mathbb{R} \times \mathbb{Z} \times \mathbb{R}_{+}$endowed with the $\sigma$-algebra and the probability measure induced by a Poisson Point Process of intensity 2 on $\mathbb{R} \times \mathbb{Z} \times \mathbb{R}_{+}$. For all $\omega \in \Omega$, for all admissible height function $\varphi \in \Gamma$ and for all $(x, y, t) \in \mathbb{R} \times \mathbb{Z} \times \mathbb{R}_{+}$, we define

$$
h(x, y, t ; \varphi, \omega)
$$

as the height function at time $t$ obtained by applying the Gates-Westcott dynamic described in the previous section with initial height profile $\varphi$ and creations $\omega$. Let us also define the continuous state-space

$$
\bar{\Gamma}:=\left\{f \in \mathcal{C}\left(\mathbb{R}^{2}\right), \forall x \in \mathbb{R}, \forall y_{1} \leq y_{2} \in \mathbb{R}, f\left(x, y_{2}\right)-f\left(x, y_{1}\right) \in\left[-\left(y_{2}-y_{1}\right), 0\right]\right\} .
$$

Notice that a continuously differentiable function on $\mathbb{R}^{2}$ is in $\bar{\Gamma}$ if and only if its gradient takes values in $\mathbb{R} \times[-1,0]$.

Theorem 3.1. Let $\left(\varphi_{n}\right)_{n \in \mathbb{N}} \in \Gamma^{\mathbb{N}}$ be a sequence of admissible initial height functions approaching a continuous function $f \in \bar{\Gamma}$ in the following sense:

$$
\forall R>0 \quad \sup _{|x|,|y| \leq R}\left|\frac{1}{n} \varphi_{n}(n x,\lfloor n y\rfloor)-f(x, y)\right| \underset{n \rightarrow \infty}{\longrightarrow} 0 .
$$

Then, for almost all $\omega$ in $\Omega$,

$$
\forall T>0 \quad \forall R>0 \quad \sup _{|x|,|y| \leq R, t \in[0, T]}\left|\frac{1}{n} h\left(n x,\lfloor n y\rfloor, n t ; \varphi_{n}, \omega\right)-u(x, y, t)\right| \underset{n \rightarrow \infty}{\longrightarrow} 0,
$$

where $u$ is the unique viscosity solution of the Hamilton-Jacobi equation

$$
\left\{\begin{aligned}
\partial_{t} u & =v(\nabla u) \\
u(\cdot, \cdot, 0) & =f
\end{aligned}\right.
$$


with

$$
v\left(\rho_{1}, \rho_{2}\right)=\frac{1}{\pi} \sqrt{\pi^{2} \rho_{1}^{2}+4 \sin ^{2}\left(\pi \rho_{2}\right)} .
$$

Remark 3.2. For any continuous function $f \in \bar{\Gamma}$, we can always find a sequence of functions $\varphi_{n} \in \Gamma$ approaching $f$ in the sense of (3.3) as we will show later in Proposition 4.10.

Before proving this theorem, we will remind the definition of the viscosity solution of Hamilton-Jacobi equations in the next section, explain why it is unique and state sufficient conditions to identify it. In Section 3.3, we will present useful results about equilibrium measures taken from $[19,20]$, where the speed function in (3.6) is also computed.

\subsection{Viscosity solutions of Hamilton-Jacobi equations}

In this section, we briefly recall some elements of the theory of Hamilton-Jacobi Partial Differential Equations. In order to show Theorem 3.1, all we need to know about viscosity solutions is gathered in Theorem 3.4 and in Proposition 3.5. The interested reader can find more background and motivations about Hamilton-Jacobi equations in the monography [7] for instance.

Given $f, v \in \mathcal{C}\left(\mathbb{R}^{d}\right)$, we consider the following first order PDE:

$$
\left\{\begin{aligned}
\partial_{t} u & =v(\nabla u) & & \text { on } \mathbb{R}^{d} \times(0,+\infty) \\
u(\cdot, 0) & =f & & \text { on } \mathbb{R}^{d} .
\end{aligned}\right.
$$

Under some further regularity conditions on $v$ and $f$, it is possible to apply the method of characteristics to obtain a local classical solution. In general, whatever the regularity of $v$ and $f$, shocks for $\nabla u$ appear in finite time and the solution is no more differentiable. In order to give a definition of solution that is global in time, we introduce the classical concept of viscosity solution that guarantees existence and uniqueness under suitable assumptions.

Definition 3.3. We say that $u: \mathbb{R}^{d} \times[0, T] \rightarrow \mathbb{R}$ is a viscosity solution of (3.7) on $\mathbb{R}^{d} \times[0, T]$ if $u$ is continuous, $u(., 0)=f$ and $u$ is both a subsolution and a supersolution.

A function $u$ is a subsolution (respectively a supersolution) if for all $\phi \in \mathcal{C}^{\infty}\left(\mathbb{R}^{d} \times(0, T)\right)$ and all $\left(x_{0}, t_{0}\right) \in \mathbb{R}^{d} \times(0, T)$ such that $\phi\left(x_{0}, t_{0}\right)=u\left(x_{0}, t_{0}\right)$ and $\phi \geq u$ (resp. $\phi \leq u$ ) on a neighbourhood of $\left(x_{0}, t_{0}\right)$, the following inequality holds:

$$
\begin{aligned}
\partial_{t} \phi\left(x_{0}, t_{0}\right) & \leq v\left(\nabla \phi\left(x_{0}, t_{0}\right)\right) \\
\left(\operatorname{resp} . \partial_{t} \phi\left(x_{0}, t_{0}\right)\right. & \left.\geq v\left(\nabla \phi\left(x_{0}, t_{0}\right)\right)\right) .
\end{aligned}
$$

We won't address the question of general existence of viscosity solutions because, in our case, we will show existence by proving that the hydrodynamic limit is indeed a solution of (3.5). However, a result of uniqueness will be needed to identify the potential limit points. The following Theorem shown by Ishii can be obtained as a corollary of [13, Th. 2.5].

Theorem 3.4. If $v$ is globally Lipschitz, there is at most one viscosity solution of (3.7) on $\mathbb{R}^{d} \times[0, T]$.

Since the function $v$ in (3.6) is globally Lipschitz, there is at most one viscosity solution of (3.5).

The next proposition gives sufficient conditions to identify the viscosity solution of (3.5). Even if it is stated for the special case of functions living in the two-dimensional continuous state-space $\bar{\Gamma}$ defined in (3.2) and for the speed function $v$ defined in (3.6), it can be easily extended to a more general framework. 
Proposition 3.5. Let $T$ be a positive real number and $S(s, t, \cdot)_{0 \leq s \leq t \leq T}$ be a family of functions from $\bar{\Gamma}$ into itself satisfying the following properties:

1. Translation invariance: for all $f \in \bar{\Gamma}$, all $c \in \mathbb{R}$ and all $s \leq t$,

$$
S(s, t, f+c)=S(s, t, f)+c .
$$

2. Monotonicity: for all $s \leq t$, and all $f, g \in \bar{\Gamma}$,

$$
f \leq g \Rightarrow S(s, t, f) \leq S(s, t, g) .
$$

3. Locality: There exists $\alpha>1$ such that for all $f, g \in \bar{\Gamma}$, all $s \leq t$, all $x \in \mathbb{R}^{2}$ and all $R \geq 0$

$$
\sup _{z \in \mathcal{B}(x, R)}|S(s, t, f)(z)-S(s, t, g)(z)| \leq \sup _{z \in \mathcal{B}(x, R+\alpha(t-s))}|f(z)-g(z)|,
$$

where $\mathcal{B}(x, r)$ is the ball of centre $x$ and radius $r$ for the supremum norm on $\mathbb{R}^{2}$.

4. Semi-group: for all $r \leq s \leq t$ and all $f \in \bar{\Gamma}$,

$$
S(r, t, f)=S(s, t, S(r, s, f)) \text { and } S(t, t, f)=f .
$$

5. Compatibility with linear solutions: for all linear function $f_{\rho}: x \mapsto \rho \cdot x$ with $\rho \in \mathbb{R} \times[-1,0]$ and all $s \leq t$,

$$
S\left(s, t, f_{\rho}\right)=f_{\rho}+v(\rho)(t-s) .
$$

For any $f \in \bar{\Gamma}$, if $(x, t) \mapsto S(0, t, f)(x)$ is continuous, then it is a viscosity solution of (3.7).

The proof of this proposition is postponed to Appendix A.

\subsection{Equilibrium measures}

In this section, we briefly remind a few facts about equilibrium measures, following Prähofer and Spohn [19, 20]. They identified a family of random height functions, whose spatial height differences have a law that is translation-invariant with a slope parameter $\rho$ in $\mathbb{R} \times(-1,0)$, and are stationary with respect to time (Gates and Westcott already treated the case $\rho \in\{0\} \times(-1,0)$ when they introduced their model in [11]). Prähofer and Spohn also computed the stationary growth speed $v(\rho)$ which gives the candidate speed function of the Hamilton-Jacobi equation (3.6) in Theorem 3.1 and showed that the variance of spatial height differences behaves logarithmically. To do so, they used fermionic Fock space tools to carry out a fine analysis of the equilibrium measures. Let us sum up useful results, most of which can be recovered or easily deduced from [20, Section 6] and others will be detailed in Appendix B.

The starting point of Prähofer and Spohn $[19,20]$ is the analysis of the Gates-Westcott model in a periodized setting, i.e. on a torus $[-M, M) \times \llbracket-N, N-1 \rrbracket$. Let us remark that, even though we use different notations, we follow the construction of [20] rather than [19] in which a more complicated "twisted" periodic boundary condition is considered (both constructions lead to the same results in the infinite volume limit of the torus). The allowed height profiles have space gradients that are periodic with horizontal period $2 \mathrm{~N}$ and vertical period $2 M$. They evolve according to the periodised Gates-Westcott dynamic i.e the Gates-Wescott dynamic with periodised Poissonian creations $[\omega]^{M, N}$ defined from $\omega$ as follows:

$$
(x, y, t) \in[\omega]^{M, N} \Leftrightarrow\left([x]^{M},[y]^{N}, t\right) \in \omega,
$$


where $[x]^{M}$ is the unique number in $[-M, M)$ equal to $x$ modulo $2 M$ and similarly for $[y]^{N}$. In [20, Section 6.2], the author defined a family of random height functions (see [20, equation (6.9)]) taking values in $\Gamma$, whose law is indexed by weight parameters $\eta^{ \pm}$on antikinks and on kinks and a slope parameter along $y$ (related to the density of level lines). The space gradients of these functions are $2 M, 2 N$ periodic and their law is translation invariant and time stationary. Fixing properly the weights $\eta^{ \pm}$and the line density, one can guarantee that the average slope approaches any fixed $\rho$ in $\mathbb{R} \times(-1,0)$ when the size of the torus tends to infinity (sending first $M$ and then $N$ to infinity). We call then $\varphi_{M, N, \rho}$ the stationary periodized profile with limit slope $\rho$, so that

$$
\forall(x, y) \in \mathbb{R} \times \mathbb{Z} \quad \lim _{N \rightarrow \infty} \lim _{M \rightarrow \infty} \mathbb{E}\left[\varphi_{M, N, \rho}(x, y)\right]=\rho \cdot(x, y)
$$

(we are fixing here $\varphi_{M, N, \rho}(0,0)=0$ ) and stationarity translates into

$$
\forall t \geq 0 \quad h\left(\cdot, \cdot, t ; \varphi_{M, N, \rho},[\omega]^{M, N}\right)-h\left(0,0, t ; \varphi_{M, N, \rho},[\omega]^{M, N}\right) \stackrel{\text { law }}{=} \varphi_{M, N, \rho}(\cdot, \cdot) .
$$

In $[19,20]$, the authors showed that the joint probability density of kinks, antikinks and occupation variables (i.e the set of $(x, y)$ such that $\varphi_{M, N, \rho}(x, y+1)-\varphi_{M, N, \rho}(x, y)=-1$ ) has a determinantal structure and identified the associated kernel. When the size of the torus tends to infinity, the expression of this kernel somehow simplifies (see [20, equation (6.20)]). Also, the average growth velocity is equal to the sum of the kink and antikink densities (independent of time by stationarity), and one obtains [20, Equation (6.24)]:

$$
\forall(x, y, t) \in \mathbb{R} \times \mathbb{Z} \times \mathbb{R}_{+} \quad \lim _{N \rightarrow \infty} \lim _{M \rightarrow \infty} \mathbb{E}\left[h\left(x, y, t ; \varphi_{M, N, \rho},[\omega]^{M, N}\right)\right]=\rho \cdot(x, y)+v(\rho) t,
$$

with $v(\rho)$ as in (3.6).

Prähofer and Spohn also computed the covariance (or "structure function") between kinks, antikinks and occupation variables (see [20, Equation (6.30)] and [19, Equation (27) and (29)]). They deduced that, after taking the infinite volume limit, the variance of the height difference at equilibrium is equivalent to $\pi^{-2} \log (\|(x, y)\|)$ as $\|(x, y)\| \rightarrow \infty$, but under the technical constraint that $y / x$ is constant or $x=\mathrm{o}(y)$. For our purposes, we will simply need the following upper bound that holds without technical restriction on $x, y$ :

$$
\lim _{N \rightarrow \infty} \lim _{M \rightarrow \infty} \operatorname{Var}\left(\varphi_{M, N, \rho}(x, y)\right)=\underset{\|(x, y)\| \rightarrow \infty}{\mathrm{O}}(\log (\|(x, y)\|)) .
$$

Equation (3.13) can be is easily shown by bounding the variance of $\varphi_{M, N, \rho}(x, y)$ by twice the sum of the variance of $\varphi_{M, N, \rho}(x, 0)$ and the variance of $\varphi_{M, N, \rho}(x, y)-\varphi_{M, N, \rho}(x, 0)$ (by Cauchy-Schwarz inequality) which grow logarithmically w.r.t $|x|$ and $|y|$, according to the asymptotic computations of Prähofer and Spohn.

Finally, it can be shown that the kink/antikink covariance decays like the inverse of the distance squared multiplied by a bounded oscillating term (an upper bound will be proven in Appendix B). Note that this is similar to the large-distance behavior of dimerdimer correlations in dimer models [15]. From this, it is easy deduce (see Appendix B) that

$$
\lim _{N \rightarrow \infty} \lim _{M \rightarrow \infty} \operatorname{Var}\left(N_{M, N, \rho}^{ \pm}\left(\Lambda_{R}\right)\right)=\underset{R \rightarrow \infty}{\mathrm{O}}\left(R^{2} \log R\right),
$$

where $N_{M, N, \rho}^{ \pm}\left(\Lambda_{R}\right)$ is the number of antikinks/kinks of $\varphi_{M, N, \rho}$ in the domain $\Lambda_{R}:=$ $[-R, R] \times \llbracket-R, R \rrbracket$.

\section{Strategy of proof of Theorem 3.1}

The crucial point is Proposition 3.5 which gives sufficient conditions for identifying the viscosity solution of (3.5). Most of these conditions are naturally satisfied by the 
microscopic Gates-Westcott dynamics, apart from the "compatibility with linear solutions" which requires a study of the process started from the translation invariant stationary measures, beyond what was obtained in $[19,20]$. The rest of the proof is based on compactness arguments, that allow to show sub-sequential existence of $S(s, t, \cdot)$ as the limit of the random microscopic semi-group $S_{n}(s, t, \cdot, \omega)$, associated to the rescaled GatesWestcott dynamics. At the end, one identifies the limiting continuous semi-group thanks to Proposition 3.5. The main steps of the proof are summed up as follows:

1. Construction of a sequence of random discrete semi-groups $\left(S_{n}(s, t ; ., \omega)\right)_{0 \leq s \leq t, n \in \mathbb{N}}$ (that will be defined more precisely in Section 4.2, Definition 4.9):

$$
S_{n}(s, t ; ., \omega):\left\{\begin{array}{l}
\bar{\Gamma} \longrightarrow \mathcal{F}\left(\mathbb{R}^{2}\right) \\
f \longmapsto \frac{1}{n} h\left(n .,\lfloor n .\rfloor, n(t-s) ; \varphi_{n}^{f}, \tau_{n s} \omega\right),
\end{array}\right.
$$

with $\varphi_{n}^{f} \in \Gamma$ approaching $f$ in the sense of (3.3): $\left\|\frac{1}{n} \varphi_{n}^{f}(n .,\lfloor n .\rfloor)-f\right\|_{\infty} \leq 2 / n, \tau_{n s} \omega$ is the time translation by $-n s$ of $\omega$ defined later in (4.6) and where $\mathcal{F}\left(\mathbb{R}^{2}\right)$ is the set of functions from $\mathbb{R}^{2}$ to $\mathbb{R}$. The function $S_{n}(s, t, f ; \omega)$ should be thought of as the rescaled height function following the dynamic starting close from the continuous initial profile $f$ and with Poissonian creations taken between the macroscopic times $s$ and $t$.

2. Compactness: Show that there exists a subset $\Omega_{0} \subseteq \Omega$ of probability 1 such that for any fixed $\omega \in \Omega_{0}$, from any subsequence $\left(n_{k}\right)_{k \in \mathbb{N}}$, we can extract a subsubsequence $\left(n_{k_{l}}\right)_{l \in \mathbb{N}}$ such that for any function $f \in \bar{\Gamma},\left(S_{n_{k_{l}}}(\cdot, \cdot ; f, \omega)\right)_{l \in \mathbb{N}}$ (seen as a sequence of functions from $\left\{(s, t) \in[0, T]^{2}, s \leq t\right\}$ to $\mathcal{F}\left(\mathbb{R}^{2}\right)$ ) converges for the topology of uniform convergence on all compact sets to a certain limiting function $S(\cdot, \cdot ; f, \omega)$ which is continuous in space and time. The proof relies on a control of spatiotemporal height differences and on an adaptation of Arzelà-Ascoli's Theorem (see Proposition D.1).

3. Identification of the limit: Show that any such limit $S(., . ; ., \omega)$ satisfies the sufficient conditions of Proposition 3.5 thus $(x, y, t) \mapsto S(0, t ; f, \omega)(x, y)$ is the unique viscosity solution of (3.5). The knowledge on equilibrium measures explained in Section 3.3 will be used to show compatibility with linear solutions.

\section{Construction of a sequence of random discrete semi-groups}

Let us start by defining, for later use, the set of creations that lead to an actual height increase.

Definition 4.1. For all $\omega \in \Omega$ and all $\varphi \in \Gamma$, we define the subset of effective creations:

$$
\omega^{\varphi}:=\left\{(x, y, t) \in \omega: h(x, y, t ; \varphi, \omega)-h\left(x, y, t^{-} ; \varphi, \omega\right)=1\right\} .
$$

It is a subset of $\omega$ that depends (non trivially) only on $\varphi$ and $\omega$. For all $y \in \mathbb{Z}$, we define the restriction of $\omega^{\varphi}$ and $\omega$ to line $y$ :

$$
\begin{aligned}
\omega_{y}^{\varphi} & :=\omega^{\varphi} \cap\left(\mathbb{R} \times\{y\} \times \mathbb{R}_{+}\right) \\
\omega_{y} & :=\omega \cap\left(\mathbb{R} \times\{y\} \times \mathbb{R}_{+}\right)
\end{aligned}
$$

By abuse of notation, we will see $\omega_{y}$ and $\omega_{y}^{\varphi}$ as subsets of $\mathbb{R}^{2}$.

\subsection{Useful properties of the microscopic dynamic}

In this section, we present useful properties satisfied by the microscopic dynamic that will be useful to apply Proposition 3.5 later on but also to show compactness. 
Lemma 4.2 (Translation invariance). For all constant $m \in \mathbb{Z}$, all $\omega \in \Omega$, all $\varphi \in \Gamma$ and all $t \in \mathbb{R}_{+}$,

$$
h(\cdot, \cdot, t ; \varphi+m, \omega)=h(\cdot, \cdot, t ; \varphi, \omega)+m .
$$

Proof. Having fixed $\omega$, by definition, the Gates-Westcott dynamic only depends on the height differences of the initial height function (kinks/antikinks and relative height differences along $y$ ). Therefore, the temporal height growth $h(\cdot, \cdot, t ; \varphi, \omega)-\varphi$ depends on $\varphi$ only through its spatial height differences hence is invariant by addition of a constant $m$ to the initial function $\varphi$.

Lemma 4.3 (Monotonicity). For all $\varphi_{1}, \varphi_{2} \in \Gamma$, for all $\omega \in \Omega$, and all $t \in \mathbb{R}_{+}$,

$$
\varphi_{1} \leq \varphi_{2} \Rightarrow h\left(., ., t ; \varphi_{1}, \omega\right) \leq h\left(., ., t ; \varphi_{2}, \omega\right) .
$$

Proof. As explained in Remark 2.3, the dynamic can be defined locally and thus it is enough to show this Lemma when there are finitely many creations. It is not hard to show that the deterministic part of the dynamic (lateral expansion and annihilation) is non-decreasing with respect to the initial condition. We just have to check that any creation preserves monotonicity.

Suppose that there is a creation at $(x, y, t)$ and that $h\left(., ., s ; \varphi_{1}, \omega\right) \leq h\left(., ., s ; \varphi_{2}, \omega\right)$ for $s<t$. Let us show that $h\left(x, y, t ; \varphi_{1}, \omega\right) \leq h\left(x, y, t ; \varphi_{2}, \omega\right)$. If $h\left(x, y, t^{-} ; \varphi_{1}, \omega\right)<$ $h\left(x, y, t^{-} ; \varphi_{2}, \omega\right)$, then there is nothing to show since the height can only jump by one after a creation. If $h\left(x, y, t^{-} ; \varphi_{1}, \omega\right)=h\left(x, y, t^{-} ; \varphi_{2}, \omega\right)$ and if the creation is allowed for the dynamic starting from $\varphi_{1}$, then so it is for the one starting from $\varphi_{2}$ because

$$
h\left(x, y-1, t^{-} ; \varphi_{2}, \omega\right)-h\left(x, y, t^{-} ; \varphi_{2}, \omega\right) \geq h\left(x, y-1, t^{-} ; \varphi_{1}, \omega\right)-h\left(x, y, t^{-} ; \varphi_{1}, \omega\right)=1
$$

and $h\left(x, y, t^{-} ; \varphi_{2}, \omega\right)-h\left(x, y+1, t^{-} ; \varphi_{2}, \omega\right) \leq h\left(x, y, t^{-} ; \varphi_{1}, \omega\right)-h\left(x, y+1, t^{-} ; \varphi_{1}, \omega\right)=0$.

In any case, the monotonicity is preserved after a creation.

For $\omega \in \Omega$, we define $\tau_{s} \omega$, the time translation by $-s$ of $\omega$ as follows:

$$
\forall(x, y, t) \in \mathbb{R} \times \mathbb{Z} \times \mathbb{R}_{+},(x, y, t) \in \tau_{s} \omega \Leftrightarrow(x, y, t+s) \in \omega .
$$

Lemma 4.4 (Markov property). For all $\varphi \in \Gamma$, all $0 \leq s \leq t$ and all $\omega \in \Omega$,

$$
h(., ., t ; \varphi, \omega)=h\left(., ., t-s ; h(., ., s ; \varphi, \omega), \tau_{s} \omega\right),
$$

and for all $0 \leq r \leq s \leq t$,

$$
h\left(., ., t-r ; \varphi, \tau_{r} \omega\right)=h\left(., ., t-s ; h\left(., ., s-r ; \varphi, \tau_{r} \omega\right), \tau_{s} \omega\right) .
$$

Proof. From Remark 2.3, we can assume that $\omega$ contains finitely many points. In this case, the first point follows directly from the construction of the dynamic. The second point is obtained from the first point applied to $\left(s^{\prime}, t^{\prime}, \omega^{\prime}\right)=\left(s-r, t-r, \tau_{r} \omega\right)$.

Next, as announced in Remark 2.3, we are going to show that the dynamic on a bounded space-time domain only depends on the initial height function and the creations on a bigger domain that grows linearly with time with high probability. To make this statement precise, for any $x \in \mathbb{R}^{2}, R \geq 0, t \in \mathbb{R}_{+}$and $\alpha>0$, let us define

$$
A_{x, R, t, \alpha}=\left\{\begin{array}{ll}
\forall \varphi, \varphi^{\prime} \in \Gamma \quad \forall \omega^{\prime} \in \Omega \\
\omega, \quad \text { if } \varphi=\varphi^{\prime} \text { on } \mathcal{B}(x, R+\alpha t) \text { and } \omega^{\prime}=\omega \text { on } \mathcal{B}(x, R+\alpha t) \times[0, t] \\
\text { then } \forall u \leq t, h\left(\cdot, \cdot, \cdot ; \varphi, \tau_{u} \omega\right)=h\left(\cdot, \cdot, \cdot ; \varphi^{\prime}, \tau_{u} \omega^{\prime}\right) \text { on } \mathcal{B}(x, R) \times[0, t-u]
\end{array}\right\}
$$

where the notation $\mathcal{B}$ abusively denotes the ball (for the supremum norm) intersected with $\mathbb{R} \times \mathbb{Z}$. 
Proposition 4.5 (Linear propagation of information). There exist constants $\alpha>1$ and $\gamma>0$, such that for all $R>0$, all $t \in \mathbb{R}_{+}$and all $x \in \mathbb{R}^{2}$,

$$
\mathbb{P}\left(A_{n x, n R, n t, \alpha}\right) \stackrel{n \rightarrow \infty}{=} 1-\mathrm{O}\left(e^{-\gamma t n}\right) .
$$

Proof. To lighten the notations, without loss of generality, we will assume that $x=0$. The idea of the proof is the following. If the height functions differ on $\mathcal{B}(0, R) \times[0, t]$ and if initial conditions and creations agrees on $\mathcal{B}(0, R+\alpha t)$, then there must exists a "chain of creations" of length at least $\alpha t$ (connecting $\mathcal{B}(0, R)$ to the complement of $\mathcal{B}(0, R+\alpha t)$ ) in a time interval of length less than $t$ (see Lemma 4.6). This is unlikely if $\alpha$ is chosen big enough and if $t$ goes to infinity.

Lemma 4.6. Let $\varphi, \varphi^{\prime} \in \Gamma$ agreeing on $\mathcal{B}(0, R+\alpha t)$ and $\omega, \omega^{\prime} \in \Omega$ agreeing on $\mathcal{B}(0, R+$ $\alpha t) \times[0, t]$. If $h(\cdot, \cdot, \cdot, \varphi ; \omega)$ and $h\left(\cdot, \cdot, \cdot, \varphi^{\prime} ; \omega^{\prime}\right)$ differ on $\mathcal{B}(x, R) \times[0, t]$, then, there must exist a sequence $\left(x_{i}, y_{i}, t_{i}\right)_{0 \leq i \leq k}$ with $k:=\lfloor\alpha t\rfloor$ satisfying

- $\left|y_{0}\right| \leq R$ and $\left|y_{i+1}-y_{i}\right| \leq 1$, for all $i \in \llbracket 0, k-1 \rrbracket$,

- $0 \leq t_{k} \leq \cdots \leq t_{0} \leq t$ and $\left(x_{i}, t_{i}\right) \in \omega_{y_{i}}$ for all $i \in \llbracket 0, k \rrbracket$, with $\omega_{y}$ as in Definition (4.1)

- $\left|x_{0}\right| \leq R+t-t_{0}$ and $\left|x_{i+1}-x_{i}\right| \leq t_{i}-t_{i+1}$ for all $i \in \llbracket 0, k-1 \rrbracket$.

Before proving this Lemma, let us finish the proof of Proposition 4.5. If $\omega \notin$ $A_{0, R, t, \alpha}$, then there must exists $\varphi, \varphi^{\prime}, \omega^{\prime}$ as in Lemma 4.6 and some $u \in[0, t]$ such that $h\left(\cdot, \cdot, \cdot, \varphi ; \tau_{u} \omega\right)$ and $h\left(\cdot, \cdot, \cdot, \varphi^{\prime} ; \tau_{u} \omega^{\prime}\right)$ differ on $\mathcal{B}(x, R) \times[0, t-u]$. By applying Lemma 4.6 at time $t-u$ and with creations $\tau_{u} \omega$ and $\tau_{u} \omega^{\prime}$ we get a chain of creations $\left.\left(x_{i}, y_{i}, t_{i}\right)_{0 \leq i \leq\lfloor\alpha t}\right\rfloor$ such that $\left(x_{i}, y_{i}, t_{i}+u\right)_{0 \leq i \leq\lfloor\alpha t\rfloor}$ satisfies the 3 points in Lemma 4.6. In order to be consistent with the definition of $C^{\uparrow}$ in Appendix $C$, we relabel this sequence by setting $\left(x_{i}^{\prime}, y_{i}^{\prime}, t_{i}^{\prime}\right)_{0 \leq i \leq\lfloor\alpha t\rfloor}:=\left(x_{\lfloor\alpha t\rfloor-i}, y_{\lfloor\alpha t\rfloor-i}, t_{\lfloor\alpha t\rfloor-i}+u\right)_{0 \leq i \leq\lfloor\alpha t\rfloor}$ so that $\left|x_{i+1}^{\prime}-x_{i}^{\prime}\right| \leq t_{i+1}^{\prime}-t_{i}^{\prime}$. Doing this, we see that

$$
{ }^{c} A_{0, R, t, \alpha} \subseteq \bigcup_{\underline{y}^{\prime} \in \mathcal{Y}_{R,\lfloor\alpha t\rfloor}} C_{\omega, \underline{y}^{\prime}}^{\uparrow}\left(T_{R, t}\right)
$$

where $\mathcal{Y}_{R, n}$ is defined by $\mathcal{Y}_{R, n}:=\left\{\left(y_{0}^{\prime} \cdots y_{n}^{\prime}\right) \in \mathbb{Z}^{n+1},\left|y_{n}^{\prime}\right| \leq R, \forall i \in \llbracket 0, n-1 \rrbracket\left|y_{i+1}^{\prime}-y_{i}^{\prime}\right| \leq\right.$ $1\}$ and $T_{R, t}$ is the trapezoid defined by $T_{R, t}:=\{(x, s), s \in[0, t],|x| \leq R+t-s\}$. By

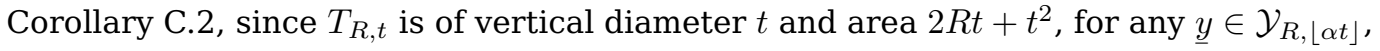

$$
\mathbb{P}\left(C_{\omega, \underline{y}}^{\uparrow}\left(T_{R, t}\right)\right) \leq 2\left(2 R t+t^{2}\right)\left(\frac{4 e^{2} t^{2}}{\lfloor\alpha t\rfloor^{2}}\right)^{\lfloor\alpha t\rfloor} .
$$

Now, since $\mathcal{Y}_{R,\lfloor\alpha t\rfloor}$ is of cardinality bounded by $(2\lfloor R\rfloor+1) 3^{\lfloor\alpha t\rfloor}$, by union bound,

$$
\mathbb{P}\left({ }^{c} A_{0, R, t, \alpha}\right) \leq 2\left(2 R t+t^{2}\right)(2\lfloor R\rfloor+1)\left(\frac{12 e^{2} t^{2}}{\lfloor\alpha t\rfloor^{2}}\right)^{\lfloor\alpha t\rfloor},
$$

and thus, for $\alpha:=\sqrt{24} e$ we get

$$
\mathbb{P}\left({ }^{c} A_{0, n R, n t, \alpha}\right) \stackrel{n \rightarrow \infty}{=} \mathrm{O}\left(n^{3} 2^{-\alpha n t}\right),
$$

and the proof of Proposition 4.5 is concluded by choosing any $\gamma<\alpha \ln 2$.

Proof of Lemma 4.6. Let us introduce some notations (we will also use the notation $\omega^{\varphi}, \omega_{y}^{\varphi}$ as in Definition 4.1). For all $(x, t) \in \mathbb{R} \times \mathbb{R}_{+}$, we define $C_{x, t}^{-}:=\{(z, s) \in \mathbb{R} \times$ $\left.\mathbb{R}_{+},|z-x| \leq t-s\right\}$. By speed one propagation of kinks/antikinks, $h(x, y, t, \varphi ; \omega)$ only depends on $\omega_{y}^{\varphi} \cap C_{x, t}^{-}$and on $\varphi(z, y)$ for $z \in[x-t, x+t]$. This fact can also be seen as a consequence of Lemma 5.3 below. Now, we are going to construct by induction a chain of creations like in Lemma 4.6. 
Construction of $\left(x_{0}, y_{0}, t_{0}\right)$ : Assume that there exists $(x, y, s) \in \mathcal{B}(0, R) \times[0, t]$ such that we have $h(x, y, s, \varphi ; \omega) \neq h\left(x, y, s, \varphi^{\prime} ; \omega^{\prime}\right)$. Let us fix such a $(x, y, s)$ and set $y_{0}:=y$. By the discussion above, since $\varphi\left(\cdot, y_{0}\right)$ and $\varphi^{\prime}\left(\cdot, y_{0}\right)$ agree on the interval $[-R-\alpha t, R+\alpha t] \supseteq$ $[x-t, x+t]$ (because $|x| \leq R$ and $\alpha>1$ ), necessarily $\omega_{y_{0}}^{\varphi} \cap C_{x, s}^{-}$and $\left(\omega^{\prime}\right)_{y_{0}}^{\varphi^{\prime}} \cap C_{x, s}^{-}$are distinct. In other words, we can find $\left(x_{0}, t_{0}\right) \in \omega_{y_{0}} \cap C_{x, s}^{-}\left(=\omega_{y_{0}}^{\prime} \cap C_{x, s}^{-}\right.$by assumption on $\left.\omega^{\prime}\right)$ corresponding to a kink/antikink creation that occurs for one of the dynamics but not for both (and such that $\left|x_{0}\right| \leq R+t-t_{0}$ ). Consequently, the height functions must differ either at $\left(x_{0}, y_{0}-1, t_{0}^{-}\right),\left(x_{0}, y_{0}, t_{0}^{-}\right)$or $\left(x_{0}, y_{0}+1, t_{0}^{-}\right)$(otherwise the creation would have been accepted or rejected simultaneously in both dynamics).

Construction of $\left(x_{i+1}, y_{i+1}, t_{i+1}\right)$ : According to the three possibilities above, we set $y_{1}$ to be equal to $y_{0}-1, y_{0}$ or $y_{0}+1$ (respectively in the first, second and third possibility). If $y_{1}$ is still in $\mathcal{B}(0, R+\alpha t)$, we can repeat the procedure above and find some $\left(x_{1}, t_{1}\right) \in$ $\omega_{y_{1}} \cap C_{x_{0}, t_{0}}^{-}$(hence $\left|x_{1}-x_{0}\right| \leq t_{0}-t_{1}$ ) corresponding to a creation that occurs for one of the dynamic but not for both and so on. This construction continues as long as $y_{i} \in \mathcal{B}(0, R+\alpha t)$ and note that $y_{i}$ cannot exit $\mathcal{B}(0, R+\alpha t)$ for $i \leq\lfloor\alpha t\rfloor$. Overall, we constructed a sequence as in Lemma 4.6. Its length is at least $\lfloor\alpha t\rfloor+1$.

Now, let us show a Lemma that relates the linear propagation of information with a Lipschitz property with respect to the initial height profile.

Lemma 4.7. For all $R \geq 0$, all $s \leq t$, all $x \in \mathbb{R}^{2}$ and all $n \in \mathbb{N}$, the following event happens with probability $1-\mathrm{O}\left(e^{-\gamma n}\right)$ as $n$ goes to infinity (with $\gamma$ as in Proposition 4.5):

$$
\sup _{z \in n \mathcal{B}(x, R)}\left|h\left(z, v-u ; \varphi, \tau_{u} \omega\right)-h\left(z, v-u ; \varphi^{\prime}, \tau_{u} \omega\right)\right| \leq \sup _{z \in n \mathcal{B}(x, R+\alpha(t-s))}\left|\varphi(z)-\varphi^{\prime}(z)\right|,
$$

for every $\varphi, \varphi^{\prime} \in \Gamma$ and every $u, v$ such that $n s \leq u \leq v \leq n t$ (and with $\alpha$ as in Proposition 4.5).

Proof. By time translation invariance of the law of the Poisson process, we can assume that $s=0$. We are going to show that the event $A_{n x, n R, n t, \alpha}$ is included in the event in the l.h.s. of (4.11). To do this, let us fix $\omega \in A_{n x, n R, n t, \alpha}, \varphi, \varphi^{\prime} \in \Gamma$ and $0 \leq u \leq v \leq n t$. We set

$$
m=\sup _{z \in n \mathcal{B}(x, R+\alpha t)}\left|\varphi(z)-\varphi^{\prime}(z)\right| \in \mathbb{N}
$$

and $\tilde{\varphi}:=\varphi \vee\left(\varphi^{\prime}+m\right)$. It is not hard to show that $\tilde{\varphi} \in \Gamma$. Now, for all $z \in n \mathcal{B}(x, R)$,

$$
\begin{aligned}
h\left(z, v-u ; \varphi, \tau_{u} \omega\right) \leq h\left(z, v-u ; \tilde{\varphi}, \tau_{u} \omega\right) & \text { by Lemma } 4.3 \text { since } \varphi \leq \tilde{\varphi} \\
=h\left(z, v-u ; \varphi^{\prime}+m, \tau_{u} \omega\right) & \omega \in A_{n x, n R, n t, \alpha} \text { and } \tilde{\varphi}=\varphi^{\prime}+m \text { on } n \mathcal{B}(x, R+\alpha t) \\
=h\left(z, v-u ; \varphi^{\prime}, \tau_{u} \omega\right)+m & \text { by Lemma 4.2. }
\end{aligned}
$$

We can prove the other inequality by exchanging $\varphi$ and $\varphi^{\prime}$ which concludes this proof.

Let us conclude this section by the next corollary which will be very useful later on.

Corollary 4.8 (Asymptotic locality). There exists $\alpha>1$ and a subset $\Omega_{0} \subseteq \Omega$ of probability 1 such that for all $\omega \in \Omega_{0}, x \in \mathbb{R}^{2}, R \geq 0,(s, t) \in \mathbb{R}^{2}$ with $0 \leq s \leq t$, there exists $N(\omega) \in \mathbb{N}$ such that for all $n \geq N(\omega)$ and all $\varphi, \varphi^{\prime} \in \Gamma$ :

$$
\begin{array}{r}
\sup _{s \leq u \leq v \leq t}\left\|h\left(n \cdot,\lfloor n \cdot\rfloor, n(v-u) ; \varphi, \tau_{n u} \omega\right)-h\left(n \cdot,\lfloor n \cdot\rfloor, n(v-u) ; \varphi^{\prime}, \tau_{n u} \omega\right)\right\|_{\infty}^{\mathcal{B}(x, R)} \\
\leq\left\|\varphi(n \cdot,\lfloor n \cdot\rfloor)-\varphi^{\prime}(n \cdot,\lfloor n \cdot\rfloor)\right\|_{\infty}^{\mathcal{B}(x, R+\alpha(t-s))}
\end{array}
$$

The proof follows easily from Lemma 4.7, Borel-Cantelli Lemma and rational approximation (up to choosing an $\alpha$ slightly larger than the one in Lemma 4.7). 


\subsection{Definition of the sequence of random discrete semi-groups}

We are going to define a sequence of functions $\left(S_{n}(s, t, f, \omega)\right)_{n \in \mathbb{N}}$ describing the rescaled dynamic, between times $s$ and $t$ and Poissonian creations $\omega$, starting at time $s$ from an initial height profile close to a continuous function $f$.

Definition 4.9. For all $\omega \in \Omega$ and $(s, t) \in \mathcal{T}:=\left\{(s, t) \in[0, T]^{2}, s \leq t\right\}$, we define

$$
S_{n}(s, t ; ., \omega): \begin{cases}\bar{\Gamma} & \longrightarrow \mathcal{F}\left(\mathbb{R}^{2}\right) \\ f & \longmapsto \frac{1}{n} h\left(n \cdot,\lfloor n \cdot\rfloor, n(t-s) ; \varphi_{n}^{f}, \tau_{n s} \omega\right),\end{cases}
$$

where $\mathcal{F}\left(\mathbb{R}^{2}\right)$ denotes the set of functions from $\mathbb{R}^{2}$ to $\mathbb{R}, \tau_{\text {ns }}$ is the temporal translation defined in (4.6) and $\varphi_{n}^{f}$ is the height profile in $\Gamma$ approaching $f$ as in the following Proposition 4.10.

Proposition 4.10. For all $n \in \mathbb{N}$, there exists a mapping

$$
\left\{\begin{array}{lc}
\bar{\Gamma} \longrightarrow & \Gamma \\
f \longmapsto & \varphi_{n}^{f}
\end{array}\right.
$$

satisfying that for all $c \in \mathbb{R}$ and $f \in \bar{\Gamma}, \varphi_{n}^{f+n^{-1}\lfloor n c\rfloor}=\varphi_{n}^{f}+\lfloor n c\rfloor$, and such that

$$
\sup _{x, y \in \mathbb{R}^{2}}\left|\frac{1}{n} \varphi_{n}^{f}(n x,\lfloor n y\rfloor)-f(x, y)\right| \leq \frac{2}{n},
$$

Therefore, the sequence of functions $\left(\varphi_{n}^{f}\right)_{n \in \mathbb{N}}$ approaches $f$ in the sense of (3.3).

Remark 4.11. We cannot just choose $\varphi_{n}^{f}:=(x, y) \mapsto\left\lfloor n f\left(n^{-1} x, n^{-1} y\right)\right\rfloor$ because it could possibly have an accumulation point of discontinuities if $f$ oscillates too much; this would violate the first condition in Definition 2.1.

Proof. For any fixed $y \in \mathbb{Z}$, we are going to define $\varphi_{n}^{f}(\cdot, y)$ as piecewise constant on $\mathbb{R}_{+}$(we will construct it similarly on $\mathbb{R}_{-}$). Let us define inductively $X_{0}^{y}=0, \varphi_{n}^{f}(0, y):=$ $\lfloor n f(0, y / n)\rfloor$ and

$$
\begin{aligned}
& \left.X_{i+1}^{y}:=\inf \left\{x \geq X_{i}^{y},\left|n f(x / n, y / n)-\varphi_{n}^{f}\left(X_{i}^{y}, y\right)\right| \geq 1\right\} \quad \text { (with } \inf \emptyset=+\infty\right) \\
& \varphi_{n}^{f}(\cdot, y)=\varphi_{n}^{f}\left(X_{i}^{y}, y\right) \quad \text { on }\left(X_{i}^{y}, X_{i+1}^{y}\right) \\
& \varphi_{n}^{f}\left(X_{i+1}^{y}, y\right)=n f\left(X_{i+1}^{y} / n, y / n\right) .
\end{aligned}
$$

By induction and by continuity of $f, \varphi_{n}^{f}\left(X_{i}^{y}, y\right) \in \mathbb{Z}$ for all $i$. Still by continuity, $X_{i+1}^{y}>X_{i}^{y}$ and $\left\{X_{i}^{y}, i \in \mathbb{N}\right\}$ is a locally finite subset of $\mathbb{R}_{+}$with $\lim _{i \rightarrow \infty} X_{i}^{y}=+\infty$. Similarly, we construct $\varphi_{n}^{f}(\cdot, y)$ on negative real numbers. Up to modifying the value at discontinuity points, we obtain a function $\varphi_{n}^{f}(\cdot, y)$ which satisfies point 1 of Definition 2.1 and which satisfies the translation invariance property $\varphi_{n}^{f+n^{-1} c}=\varphi_{n}^{f}+c$ for all $c \in \mathbb{Z}$ by construction. Moreover, by construction, for all $(x, y) \in \mathbb{R} \times \mathbb{Z},\left|\varphi_{n}^{f}(x, y)-n f(x / n, y / n)\right| \leq 1$ and thus

$$
\begin{aligned}
& \sup _{(x, y) \in \mathbb{R}^{2}}\left|\frac{1}{n} \varphi_{n}^{f}(n x,\lfloor n y\rfloor)-f(x, y)\right| \\
& \quad \leq \sup _{(x, y) \in \mathbb{R}^{2}}\left|\frac{1}{n} \varphi_{n}^{f}(n x,\lfloor n y\rfloor)-f(x,\lfloor n y\rfloor / n)\right|+\sup _{(x, y) \in \mathbb{R}^{2}}|f(x,\lfloor n y\rfloor / n)-f(x, y)| \\
& \quad \leq \frac{1}{n}+|\lfloor n y\rfloor / n-y| \leq \frac{2}{n} \quad \text { because } f \in \bar{\Gamma} .
\end{aligned}
$$

It remains to check that $\varphi_{n}^{f}$ satisfies point 2 of Definition 2.1 and hence is in $\Gamma$. To do this, let us fix $y \in \mathbb{Z}$ and show that for all $x \geq 0, \varphi_{n}^{f}(x, y+1)-\varphi_{n}^{f}(x, y) \in\{-1,0\}$ (the case 
$x<0$ being similar). Let $x \geq 0$ and $i, j$ be the unique integers such that $X_{i}^{y} \leq x<X_{i+1}^{y}$ and $X_{j}^{y+1} \leq x<X_{j+1}^{y+1}$. By construction of $\varphi_{n}^{f}$,

$$
\begin{aligned}
\varphi_{n}^{f}(x, y) & =n f\left(X_{i}^{y} / n, y / n\right) \\
\varphi_{n}^{f}(x, y+1) & =n f\left(X_{j}^{y+1} / n,(y+1) / n\right) .
\end{aligned}
$$

There are two cases: either $X_{j}^{y+1} \in\left[X_{i}^{y}, X_{i+1}^{y}\right)$ or $X_{i}^{y} \in\left[X_{j}^{y+1}, X_{j+1}^{y+1}\right)$. Since they are similar, we will just treat the first one. By definition of $X_{i+1}^{y}$, for all $z \in\left[X_{i}^{y}, X_{i+1}^{y}\right)$ we have $\left|n f(z / n, y / n)-n f\left(X_{i}^{y} / n, y / n\right)\right|<1$ and thus

$$
\begin{aligned}
& \varphi_{n}^{f}(x, y+1)-\varphi_{n}^{f}(x, y)=n f\left(X_{j}^{y+1} / n,(y+1) / n\right)-n f\left(X_{i}^{y} / n, y / n\right) \\
& =\underbrace{n f\left(X_{j}^{y+1} / n,(y+1) / n\right)-n f\left(X_{j}^{y+1} / n, y / n\right)}_{\in[-1,0] \text { since } f \in \bar{\Gamma}}+\underbrace{n f\left(X_{j}^{y+1} / n, y / n\right)-n f\left(X_{i}^{y} / n, y / n\right)}_{\in(-1,1) \text { since } X_{j}^{y+1} \in\left[X_{i}^{y}, X_{i+1}^{y}\right)} .
\end{aligned}
$$

Finally, $\varphi_{n}^{f}(x, y+1)-\varphi_{n}^{f}(x, y) \in(-2,1) \cap \mathbb{Z}=\{-1,0\}$.

\section{Compactness}

\subsection{Control on spatio-temporal height differences}

In this section, we control the spatio-temporal gradients of the height function following the Gates-Westcott dynamic by comparison with the PNG dynamic. By construction, $(x, t) \mapsto h(x, y, t ; \varphi, \omega)$ follows the PNG dynamic (see e.g. [9, Section 2] for an introduction to the model) starting from initial condition $\varphi(\cdot, y)$ with creation locations given by $\omega_{y}^{\varphi}$ as in Definition 4.1. This simple remark allows us to use the representation of PNG model in terms of directed polymer on Poisson points (see [9, Section 3.1]). First, we need to introduce some new definitions.

Definition 5.1. For any finite set $A \subseteq \mathbb{R}^{2}$, we define $L^{\uparrow}(A)$ as the maximal number of points in $A$ that can be collected by a light-path i.e a continuous path $\gamma:[0,1] \rightarrow \mathbb{R}^{2}$ satisfying that for any $0 \leq a \leq b$, we have $\gamma(b)-\gamma(a) \in\left\{(x, t) \in \mathbb{R}^{2}|x| \leq t\right\}$.

We say that a rectangle $R \subseteq \mathbb{R}^{2}$ is a light-rectangle if its sides are parallel to the straight lines $t=x$ or $t=-x$. For any $s<t$ and $(x, s),(y, t)$ such that $|y-x| \leq t-s$, we note $R_{(x, s),(y, t)}$ the unique light-rectangle of diagonal $[(x, s),(y, t)]$.

Remark 5.2. We let the reader check that the area of $R_{(x, s),(y, t)}$ is $\left((t-s)^{2}-(y-x)^{2}\right) / 2$ and that if $\left|x^{\prime}-x\right| \leq s-s^{\prime}$ then $R_{(x, s),(y, t)} \subseteq R_{\left(x^{\prime}, s^{\prime}\right),(y, t)}$ while if $\left|y^{\prime}-y\right| \leq t^{\prime}-t$ then $R_{(x, s),(y, t)} \subseteq R_{(x, s),\left(y^{\prime}, t^{\prime}\right)}$.

The next Lemma is an easy extension to arbitrary initial conditions of the equivalence between the PNG and the directed polymers model as explained in [9, Sections 2.3 and 3.1] for special "droplet" and "flat" initial conditions (see also Figure 2).

Lemma 5.3. For all $(x, y, t) \in \mathbb{R} \times \mathbb{Z} \times \mathbb{R}_{+}$, all $\varphi \in \Gamma$ and $\omega \in \Omega$,

$$
h(x, y, t ; \varphi, \omega)=\sup _{z \in[x-t, x+t]}\left\{\varphi(z, y)+L^{\uparrow}\left(\omega_{y}^{\varphi} \cap R_{(z, 0),(x, t)}\right)\right\},
$$

and the supremum is attained for some $z \in[x-t, x+t]$.

In order to control the space gradients of the interface, we need an upper bound on $L^{\uparrow}\left(\omega_{y}^{\varphi} \cap R\right)$ (or on $L^{\uparrow}\left(\omega_{y} \cap R\right)$ since $\left.\omega_{y}^{\varphi} \subseteq \omega_{y}\right)$ for large rectangles $R$. This quantity is well studied as it is related to the length of the longest increasing subsequence of a random uniform permutation, which was shown first by Hammersley to behave like the square root of the number of Poisson points in $R$ (this is also known as Ulam's problem; see [12]). 


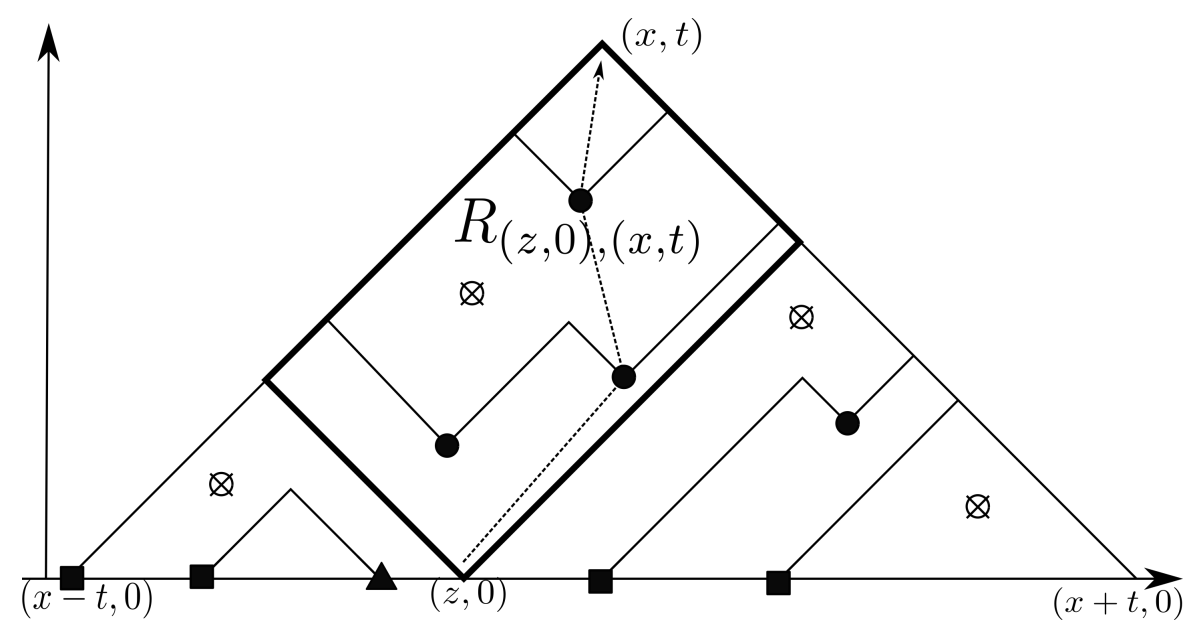

Figure 2: The graphical representation of the PNG and its interpretation in terms of Directed Polymer. The lines are drawn from the initial antikinks (triangles) and kinks (squares) of $\varphi(\cdot, y)$ and the effective creations in $\omega_{y}^{\varphi}$ (filled circles) (the creations in $\omega_{y} \backslash \omega_{y}^{\varphi}$ are marked by crossed circles and can be ignored). The height difference between two points is equal to the number of lines crossed by any light-path joining these points. A path touches at most one effective creation per line crossed and conversely, we can always find a path passing by effective creations that realises the maximum in the variational formula of Lemma 5.3.

Lemma 5.4. There exists a constant $c>0$ such that for all $\omega$ in a subset of $\Omega$ of probability 1, for all light-rectangle $R \subseteq \mathbb{R}^{2}$ and all $Y>0$,

$$
\limsup _{n \rightarrow \infty} \sup _{y \in \llbracket-n Y, n Y \rrbracket} \frac{1}{n} L^{\uparrow}\left(\omega_{y} \cap n R\right) \leq c \sqrt{\operatorname{Leb}(R)},
$$

where $\operatorname{Leb}(R)$ is the area of $R$. Therefore, up to intersecting this subset of probability 1 with $\Omega_{0}$ (defined in Corollary 4.8) we can assume that (5.2) holds for all $\omega \in \Omega_{0}$.

Proof. By Lemma C.1 in Appendix C, for all $y \in \mathbb{Z}$ and all $k \in \mathbb{N}$,

$$
\mathbb{P}\left(L^{\uparrow}\left(\omega_{y} \cap R\right) \geq k\right) \leq\left(\frac{2 e^{2} \operatorname{Leb}(R)}{k^{2}}\right)^{k} .
$$

This is a classical inequality when dealing with longest increasing subsequences that can be found for example in [24, Lemma 4.1]. Therefore, for $c=2 e$,

$$
\mathbb{P}\left(\sup _{y \in \llbracket-n Y, n Y \rrbracket}\left\{\frac{1}{n} L^{\uparrow}\left(\omega_{y} \cap n R\right)\right\} \geq c \sqrt{\operatorname{Leb}(R)}\right) \leq 2 n Y 2^{-\lceil n c \sqrt{\operatorname{Leb}(R)}\rceil} .
$$

By Borel-Cantelli Lemma, for almost all $\omega$, for any $Y>0$,

$$
\limsup _{n \rightarrow \infty} \sup _{y \in \llbracket-n Y, n Y \rrbracket} \frac{1}{n} L^{\uparrow}\left(\omega_{y} \cap n R\right) \leq c \sqrt{\operatorname{Leb}(R)} .
$$

By countability, we can have this almost surely simultaneously for all light-rectangle $R$ with rational coordinate vertices. The full proof follows by density of rational numbers and by the monotonicity with respect to inclusion of $R \mapsto L^{\uparrow}\left(\omega_{y} \cap n R\right)$.

Let us now give some consequences of Lemmas 5.3 and 5.4. 
Proposition 5.5 (Control on temporal growth). For all $\omega \in \Omega_{0}$, for all $f \in \bar{\Gamma}$, all $s \leq t$ and $(x, y) \in \mathbb{R}^{2}$,

$$
\limsup _{n \rightarrow \infty} S_{n}(s, t, f ; \omega)(x, y) \leq \sup _{|z-x| \leq t-s} f(z, y)+\sqrt{2} c(t-s),
$$

where $c$ is the same constant as in Lemma 5.4 .

Proof. By definition of $S_{n}$ and by Lemma 5.3,

$$
\begin{aligned}
& S_{n}(s, t, f ; \omega)(x, y)=\frac{1}{n} h\left(n x,\lfloor n y\rfloor, n(t-s) ; \varphi_{n}^{f}, \tau_{n s} \omega\right) \\
& =\sup _{|z-x| \leq t-s}\left\{\frac{1}{n} \varphi_{n}^{f}(n z, y)+\frac{1}{n} L^{\uparrow}\left(\left(\tau_{n s} \omega\right)_{\lfloor n y\rfloor}^{\varphi_{n}^{f}} \cap R_{(n z, 0),(n x, n(t-s))}\right)\right\} \\
& \leq \sup _{|z-x| \leq t-s}\left\{f(z, y)+\frac{2}{n}+\frac{1}{n} L^{\uparrow}\left(\left(\tau_{n s} \omega\right)_{\lfloor n y\rfloor} \cap n R_{(z, 0),(x, t-s)}\right)\right\} \quad \text { by (4.15) and } \omega^{\varphi_{n}^{f}} \subseteq \omega \\
& =\sup _{|z-x| \leq t-s}\left\{f(z, y)+\frac{2}{n}+\frac{1}{n} L^{\uparrow}\left(\omega_{\lfloor n y\rfloor} \cap n R_{(z, s),(x, s)}\right)\right\} \quad \text { by definition of } \tau_{n s} \text { in (4.6) } \\
& \leq \sup _{|z-x| \leq t-s} f(z, y)+\frac{2}{n}+\frac{1}{n} L^{\uparrow}\left(\omega_{\lfloor n y\rfloor} \cap n R_{(x, 2 s-t),(x, t)}\right) \quad \text { since } R_{(z, s),(x, t)} \subseteq R_{(x, 2 s-t),(x, t)} .
\end{aligned}
$$

We conclude the proof with Lemma 5.4 and $\operatorname{Leb}\left(R_{(x, 2 s-t),(x, t)}\right)=2(t-s)^{2}$.

Now, we establish a crucial lemma that guarantees a priori that, at any time, the asymptotic rescaled height function has at least the worst regularity between that of the initial height profile and 1/2-Hölder regularity. A posteriori, after the proof of the main theorem, we will have that it stays Lipschitz at any time if the initial condition is itself Lipschitz since this is the case for viscosity solutions of Hamilton-Jacobi equations.

Proposition 5.6 (Control on height differences along $x$ ). There exists a constant $C$ (that depends on the time horizon $T$ ) such that for all $\omega \in \Omega_{0}$, all $f \in \bar{\Gamma}$, all $(x, y) \in \mathbb{R}^{2}$, and all $\delta \in[0,1]$,

$$
\begin{aligned}
\limsup _{n \rightarrow \infty} \sup _{\substack{x_{1}, x_{2} \in[x-\delta, x+\delta] \\
0 \leq s \leq t \leq T}}\left|S_{n}(s, t, f ; \omega)\left(x_{2}, y\right)-S_{n}(s, t, f ; \omega)\left(x_{1}, y\right)\right| \\
\leq \sup _{\substack{x_{1}, x_{2} \in\left[x_{2}-\delta-T, x+\delta+T\right] \\
\left|x_{2}-x_{1}\right| \leq 2 \delta}}\left|f\left(x_{1}, y\right)-f\left(x_{2}, y\right)\right|+C \sqrt{\delta} .
\end{aligned}
$$

Proof. We start by showing the following Lemma.

Lemma 5.7. For all $y \in \mathbb{Z}$, all $x_{1}<x_{2} \in \mathbb{R}$, all $t \geq 0$, all $\varphi \in \Gamma$,

$$
\begin{aligned}
& \left|h\left(x_{2}, y, t ; \varphi, \omega\right)-h\left(x_{1}, y, t ; \varphi, \omega\right)\right| \\
& \quad \leq \sup _{\substack{z, z^{\prime} \in\left[x_{1}-t, x_{2}+t\right] \\
\left|z-z^{\prime}\right| \leq\left|x_{2}-x_{1}\right|}}\left|\varphi(z, y)-\varphi\left(z^{\prime}, y\right)\right|+\max \left(L^{\uparrow}\left(\omega_{y} \cap R_{1}\right), L^{\uparrow}\left(\omega_{y} \cap R_{2}\right)\right),
\end{aligned}
$$

with $R_{1}:=R_{\left(\frac{x_{1}+x_{2}}{2}-t,-\frac{x_{2}-x_{1}}{2}\right),\left(x_{1}, t\right)}$ and $R_{2}:=R_{\left(\frac{x_{1}+x_{2}}{2}+t,-\frac{x_{2}-x_{1}}{2}\right),\left(x_{2}, t\right)}$.

Proof. We start by showing that

$$
h\left(x_{2}, y, t ; \varphi, \omega\right)-h\left(x_{1}, y, t ; \varphi, \omega\right) \leq \sup _{z \in\left[x_{1}+t, x_{2}+t\right]}\left|\varphi(z, y)-\varphi\left(x_{1}+t, y\right)\right|+L^{\uparrow}\left(\omega_{y} \cap R_{2}\right) .
$$

By Lemma 5.3, there exists $z \in\left[x_{2}-t, x_{2}+t\right]$ such that

$$
h\left(x_{2}, y, t ; \varphi, \omega\right)=\varphi(z, y)+L^{\uparrow}\left(\omega_{y}^{\varphi} \cap R_{(z, 0),\left(x_{2}, t\right)}\right) .
$$

Two cases can occur: 
(i) If $z \in\left[x_{1}-t, x_{1}+t\right]$, then, by Lemma $5.3, h\left(x_{1}, y, t ; \varphi, \omega\right)$ is larger than $\varphi(z, y)+$ $L^{\uparrow}\left(\omega_{y}^{\varphi} \cap R_{(z, 0),\left(x_{1}, t\right)}\right)$ hence

$$
h\left(x_{2}, y, t ; \varphi, \omega\right)-h\left(x_{1}, y, t ; \varphi, \omega\right) \leq L^{\uparrow}\left(\omega_{y}^{\varphi} \cap R_{(z, 0),\left(x_{2}, t\right)}\right)-L^{\uparrow}\left(\omega_{y}^{\varphi} \cap R_{(z, 0),\left(x_{1}, t\right)}\right) .
$$

Now, for any $A, B \subseteq \mathbb{R}^{2}$ finite sets, it is not hard to show $L^{\uparrow}(A \cup B) \leq L^{\uparrow}(A)+L^{\uparrow}(B)$, and thus for any $A, B \subseteq \mathbb{R}^{2}$ finite sets,

$$
L^{\uparrow}(A)-L^{\uparrow}(B) \leq L^{\uparrow}(A \backslash B) .
$$

We apply this inequality with $A=\omega_{y}^{\varphi} \cap R_{(z, 0),\left(x_{2}, t\right)}$ and $B=\omega_{y}^{\varphi} \cap R_{(z, 0),\left(x_{1}, t\right)}$ (the creations inside the blue and red rectangles on Figure 3 ). The set $A \backslash B$ is equal to the creations inside the green rectangle which is included in the light-rectangle $R_{2}$ (surrounded by dash lines on Figure 3). Altogether, $h\left(x_{2}, y, t ; \varphi, \omega\right)-h\left(x_{1}, y, t ; \varphi, \omega\right) \leq$

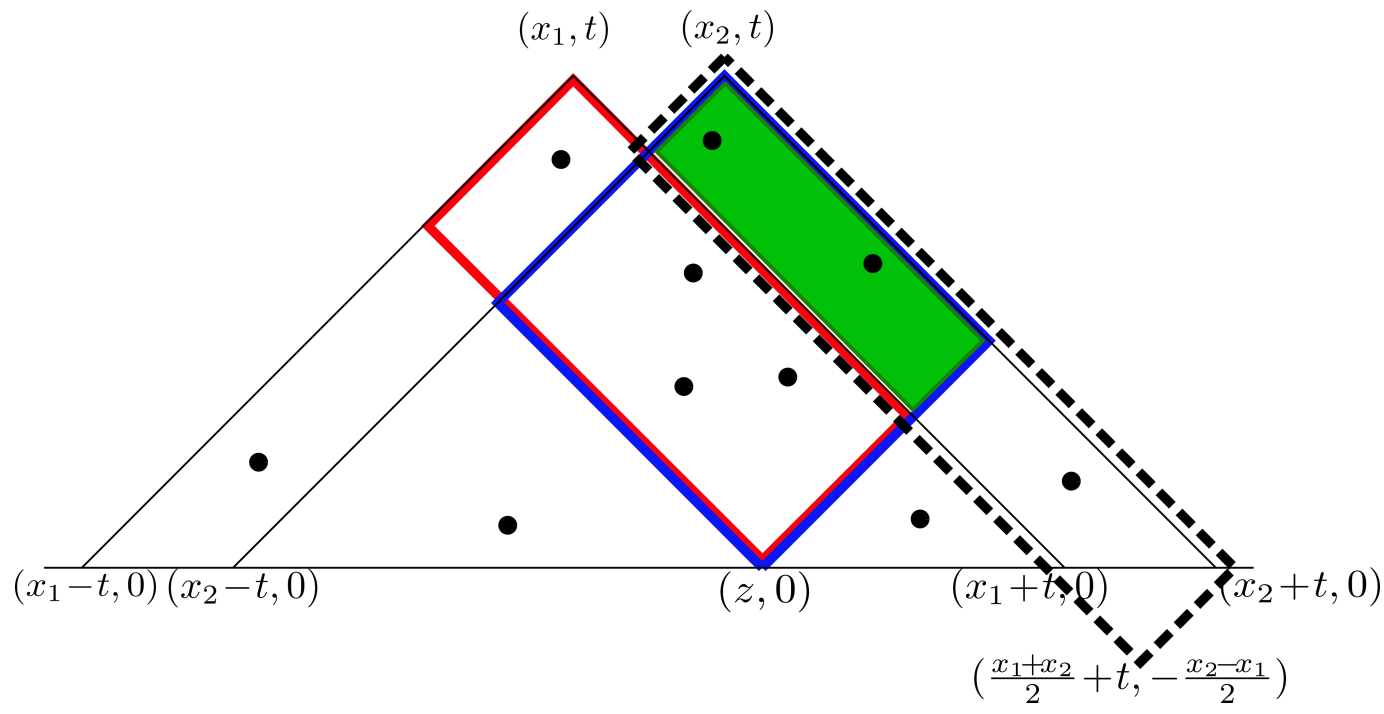

Figure 3: Illustration of the proof of Lemma 5.7. The effective creations are depicted by black circles. The blue rectangle $R_{(z, 0),\left(x_{2}, t\right)}$ and the red rectangle $R_{(z, 0),\left(x_{1}, t\right)}$ are involved in the variational formula (5.1) applied to $h\left(x_{2}, y, t\right)$ and $h\left(x_{1}, y, t\right)$. The green rectangle corresponds to the set difference of the blue and red rectangles and is included in the larger dashed-line rectangle.

$L^{\uparrow}\left(\omega_{y}^{\varphi} \cap R_{2}\right)$, which implies (5.6).

(ii) If $z \in\left[x_{1}+t, x_{2}+t\right]$, then by choosing $z^{\prime}=x_{1}+t$ in the variational formula (5.1), we get that $h\left(x_{1}, y, t ; \varphi, \omega\right) \geq \varphi\left(x_{1}+t, y\right)$ hence

$$
\begin{aligned}
h\left(x_{2}, y, t ; \varphi, \omega\right) & -h\left(x_{1}, y, t ; \varphi, \omega\right) \\
& \leq \varphi(z, y)+L^{\uparrow}\left(\omega_{y}^{\varphi} \cap R_{(z, 0),\left(x_{2}, t\right)}\right)-\varphi\left(x_{1}+t, y\right) \\
& \leq \sup _{z \in\left[x_{1}+t, x_{2}+t\right]}\left|\varphi(z, y)-\varphi\left(x_{1}+t, y\right)\right|+L^{\uparrow}\left(\omega_{y}^{\varphi} \cap R_{2}\right),
\end{aligned}
$$

since

$$
R_{(z, 0),\left(x_{2}, t\right)} \subseteq R_{\left(\frac{x_{1}+x_{2}}{2}+t,-\frac{x_{2}-x_{1}}{2}\right),\left(x_{2}, t\right)}=R_{2} \text { for all } z \in\left[x_{1}+t, x_{2}+t\right],
$$

as shown in Figure 3. This also implies (5.6). 
The proof of Lemma 5.7 is concluded by showing similarly that

$$
h\left(x_{1}, y, t ; \varphi, \omega\right)-h\left(x_{2}, y, t ; \varphi, \omega\right) \leq \sup _{z \in\left[x_{1}-t, x_{2}-t\right]}\left|\varphi(z, y)-\varphi\left(x_{2}-t, y\right)\right|+L^{\uparrow}\left(\omega_{y} \cap R_{1}\right) .
$$

Lemma 5.7 yields that for all $x-\delta \leq x_{1} \leq x_{2} \leq x+\delta$ and all $t \geq 0$,

$$
\begin{aligned}
\left|h\left(x_{2}, y, t ; \varphi, \omega\right)-h\left(x_{1}, y, t ; \varphi, \omega\right)\right| & \leq \sup _{z, z^{\prime} \in[x-\delta-t, x+\delta+t]}\left|\varphi(z, y)-\varphi\left(z^{\prime}, y\right)\right| \\
& +\max \left(L^{\uparrow}\left(\omega_{y} \cap R_{(x-t,-\delta),(x, t+\delta)}\right), L^{\uparrow}\left(\omega_{y} \cap R_{(x+t,-\delta),(x, t+\delta)}\right)\right)
\end{aligned}
$$

since one can check that

$$
R_{\left(\frac{x_{1}+x_{2}}{2}-t,-\frac{x_{2}-x_{1}}{2}\right),\left(x_{1}, t\right)} \subseteq R_{(x-t,-\delta),(x, t+\delta)}
$$

and

$$
R_{\left(\frac{x_{1}+x_{2}}{2}+t,-\frac{x_{2}-x_{1}}{2}\right),\left(x_{2}, t\right)} \subseteq R_{(x+t,-\delta),(x, t+\delta)} .
$$

Thus, for all $x_{1}, x_{2} \in[x-\delta, x+\delta]$, all $s, t$ such that $0 \leq s \leq t \leq T$, all $f \in \bar{\Gamma}$ and all $n \in \mathbb{N}$,

$$
\begin{aligned}
& \left|S_{n}(s, t, f ; \omega)\left(x_{2}, y\right)-S_{n}(s, t, f ; \omega)\left(x_{1}, y\right)\right| \\
& =\left|\frac{1}{n} h\left(n x_{2},\lfloor n y\rfloor, n(t-s), \varphi_{n}^{f}, \tau_{n s} \omega\right)-\frac{1}{n} h\left(n x_{1},\lfloor n y\rfloor, n(t-s), \varphi_{n}^{f}, \tau_{n s} \omega\right)\right| \\
& \leq \sup _{\substack{z, z^{\prime} \in[x-\delta-(t-s), x+\delta+(t-s)] \\
\left|z-z^{\prime}\right| \leq 2 \delta}}\left|\frac{1}{n} \varphi_{n}^{f}(n z,\lfloor n y\rfloor)-\frac{1}{n} \varphi_{n}^{f}\left(n z^{\prime},\lfloor n y\rfloor\right)\right| \\
& \quad+\max \left(\frac{1}{n} L^{\uparrow}\left(\omega_{\lfloor n y\rfloor} \cap n R_{(x-(t-s), s-\delta),(x, t+\delta)}\right), \frac{1}{n} L^{\uparrow}\left(\omega_{\lfloor n y\rfloor} \cap n R_{(x+(t-s), s-\delta),(x, t+\delta)}\right)\right) \\
& \leq \sup _{z, z^{\prime} \in[x-\delta-T, x+\delta+T]}^{\left|z-z^{\prime}\right| \leq 2 \delta}\left|f(z, y)-f\left(z^{\prime}, y\right)\right|+4 / n \\
& \quad+\max \left(\frac{1}{n} L^{\uparrow}\left(\omega_{\lfloor n y\rfloor} \cap n R_{(x-T,-\delta),(x, T+\delta)}\right), \frac{1}{n} L^{\uparrow}\left(\omega_{\lfloor n y\rfloor} \cap n R_{(x+T,-\delta),(x, T+\delta)}\right)\right),
\end{aligned}
$$

where the last inequality holds because of (4.15) and because $R_{(x \pm(t-s), s-\delta),(x, t+\delta)}$ is included in $R_{(x \pm T,-\delta),(x, T+\delta)}$. Note that this upper bound is uniform in $x_{1}, x_{2} \in[x-\delta, x+\delta]$ and in $s, t$ such that $0 \leq s \leq t \leq T$. We conclude the proof of Proposition 5.6 by applying Lemma 5.4 to $R_{(x \pm T,-\delta),(x, T+\delta)}$ which are of area $2\left(\delta T+\delta^{2}\right) \leq 2(T+1) \delta$ since $\delta \in[0,1]$ and by choosing $C:=c \sqrt{2(T+1)}(c$ is the same constant as in Lemma 5.4).

\subsection{Choice of the metric}

We endow $\mathcal{F}\left(\mathbb{R}^{2}\right)$ and $\bar{\Gamma} \subseteq \mathcal{C}\left(\mathbb{R}^{2}\right) \subseteq \mathcal{F}\left(\mathbb{R}^{2}\right)$ with the distance of uniform convergence on all compacts, e.g.

$$
\forall f_{1}, f_{2} \in \mathcal{F}\left(\mathbb{R}^{2}\right), d_{\infty, c}\left(f_{1}, f_{2}\right):=\sum_{i=1}^{\infty} 2^{-i}\left(\left\|f_{1}-f_{2}\right\|_{\infty}^{[-i, i]^{2}} \wedge 1\right) .
$$

For this distance, a sequence of functions $\left(f_{n}\right)_{n \in \mathbb{N}}$ converges to $f \in \mathcal{F}\left(\mathbb{R}^{2}\right)$ if and only if it converges uniformly on all compact sets of $\mathbb{R}^{2}$ to $f$.

Proposition 5.8. The metric space $\left(\mathcal{F}\left(\mathbb{R}^{2}\right), d_{\infty, c}\right)$ is complete. The metric space $\left(\bar{\Gamma}, d_{\infty, c}\right)$ is complete and separable (i.e. a Polish space). 
Proof. The completeness of $\left(\mathcal{F}\left(\mathbb{R}^{2}\right), d_{\infty, c}\right)$ is a classical fact. Since $\bar{\Gamma}$ is a closed subset of $\mathcal{C}\left(\mathbb{R}^{2}\right)$ (which is complete because closed in $\mathcal{F}\left(\mathbb{R}^{2}\right)$ and separable by approximation by polynomials with rational coefficients on any compact set) it is in turn a complete separable metric space.

Now, we denote $\mathcal{F}_{\mathcal{T}}:=\mathcal{F}\left(\mathcal{T}, \mathcal{F}\left(\mathbb{R}^{2}\right)\right.$ ) the set of functions from $\mathcal{T}$ (defined in Definition 4.9) into $\mathcal{F}\left(\mathbb{R}^{2}\right)$ which we endow with the uniform distance:

$$
\forall F_{1}, F_{2} \in \mathcal{F}_{\mathcal{T}}, D_{\infty}\left(F_{1}, F_{2}\right):=\sup _{0 \leq s \leq t \leq T} d_{\infty, c}\left(F_{1}(s, t), F_{2}(s, t)\right) .
$$

The following Proposition is standard when dealing with functional spaces with a complete set of destination such as $\mathcal{F}\left(\mathbb{R}^{2}\right)$ (by Proposition 5.8) and endowed with the uniform distance.

Proposition 5.9. The metric space $\left(\mathcal{F}_{\mathcal{T}}, D_{\infty}\right)$ is complete.

\subsection{Compactness for any fixed $\omega$ in a subset $\Omega_{0}$ of probability 1}

We recall that $\Omega_{0}$ is a subset of $\Omega$ of probability 1 introduced in Corollary 4.8 and Lemma 5.4. The goal of this section is to show the following proposition:

Proposition 5.10. For all $\omega \in \Omega_{0}$, and all sub-sequences $\left(n_{k}\right)_{k \in \mathbb{N}}$, we can extract a subsub-sequence $\left(n_{k_{l}}\right)_{l \in \mathbb{N}}$ such that for all functions $f \in \bar{\Gamma}$, the sequence $\left(S_{n_{k_{l}}}(., . ; f, \omega)\right)_{l \in \mathbb{N}}$ converges to a certain $S(\cdot, \cdot ; f, \omega)$ in $\mathcal{F}_{\mathcal{T}}$, i.e,

$$
\forall R>0, \quad \sup _{\substack{0 \leq s \leq t \leq T \\|x|,|y| \leq R}}\left|S_{n_{k_{l}}}(s, t, f ; \omega)(x, y)-S(s, t, f ; \omega)(x, y)\right| \underset{l \rightarrow \infty}{\longrightarrow} 0 .
$$

Moreover, for all $(s, t) \in \mathcal{T}, f \mapsto S(s, t ; f, \omega)$ is continuous from $\bar{\Gamma}$ into itself and for all $f \in \bar{\Gamma},(s, t) \mapsto S(s, t ; f, \omega)$ is continuous.

Proof. In all this proof, we fix $\omega \in \Omega_{0}$. Let us apply Proposition D.1 to the sequence of functions $\bar{\Gamma} \ni f \longmapsto S_{n}(\cdot, \cdot, f ; \omega) \in \mathcal{F}_{\mathcal{T}}$. From Propositions 5.8 and $5.9, \bar{\Gamma}$ is separable and $\mathcal{F}_{\mathcal{T}}$ is complete. Therefore, the proof of Proposition 5.10 follows easily from Proposition D.1 together with the next two lemmas giving asymptotic equi-continuity and pointwise relative compactness.

Lemma 5.11 (Asymptotic equi-continuity of $\left(f \mapsto S_{n}(\cdot, \cdot, f ; \omega)\right)_{n \in \mathbb{N}}$ ). For all $\omega \in \Omega_{0}$ and all $\varepsilon>0$, there exists $N \in \mathbb{N}$ such that

$$
\forall n \geq N \quad \forall f, g \in \bar{\Gamma} \quad D_{\infty}\left(S_{n}(\cdot, \cdot, f ; \omega), S_{n}(\cdot, \cdot, g ; \omega)\right) \leq 2^{\lceil\alpha T\rceil} d_{\infty, c}(f, g)+\varepsilon .
$$

The proof comes from an easy corollary of (4.12) (we will prove it in details at the end of this section).

Lemma 5.12 (Pointwise relative compactness of $\left((s, t) \mapsto S_{n}(s, t, f ; \omega)\right)_{n \in \mathbb{N}}$ in $\left.\mathcal{F}_{T}\right)$. For any $\omega \in \Omega_{0}$ and $f \in \bar{\Gamma}$, the sequence $\left((s, t) \mapsto S_{n}(s, t, f ; \omega)\right)_{n \in \mathbb{N}}$ is contained in a compact set of $\mathcal{F}_{\mathcal{T}}$. Moreover, any limit point is continuous from $\mathcal{T}$ into $\bar{\Gamma}$.

Proof of Lemma 5.12. We want to show that for any fixed $f \in \bar{\Gamma}$, from any sub-sequence of $\left((s, t) \mapsto S_{n}(s, t, f ; \omega)\right)_{n \in \mathbb{N}}$, we can find a uniformly converging sub-sub-sequence in $\mathcal{F}_{\mathcal{T}}$. We are going to apply once again Proposition D.1. The set $\mathcal{F}_{\mathcal{T}}$ is the set of functions from $\mathcal{T}$ which is compact into $\left(\mathcal{F}\left(\mathbb{R}^{2}\right), d_{\infty, c}\right)$ which is complete (by Proposition 5.8). Therefore, it is enough to show asymptotic equi-continuity and pointwise relative compactness.

Lemma 5.13 (Pointwise relative compactness of $\left(S_{n}(s, t, f ; \omega)\right)_{n \in \mathbb{N}}$ in $\mathcal{F}\left(\mathbb{R}^{2}\right)$ ). For any $\omega \in \Omega_{0}, f \in \bar{\Gamma}$ and $0 \leq s \leq t \leq T$, the sequence $\left(S_{n}(s, t, f ; \omega)\right)_{n \in \mathbb{N}}$ is contained in a compact set of $\mathcal{F}\left(\mathbb{R}^{2}\right)$. Moreover, any limit point is in $\bar{\Gamma}$. 
Proof of Lemma 5.13. As $\mathcal{F}\left(\mathbb{R}^{2}\right)$ is endowed with the topology of convergence on all compact sets of $\mathbb{R}^{2}$, it is enough to show asymptotic equi-continuity and pointwise relative compactness in order to apply Proposition D.1 once more.

1. Pointwise relative compactness: $\left(S_{n}(s, t, f ; \omega)(x, y)\right)_{n \in \mathbb{N}} \in \mathbb{R}$

By Bolzano-Weierstrass Theorem, it is enough to show that this sequence is bounded. The upper bound is a direct consequence of Proposition 5.5 while the lower bound is trivial since height functions are non-decreasing with time.

2. Asymptotic equi-continuity of $\left((x, y) \mapsto S_{n}(s, t, f ; \omega)(x, y)\right)_{n \in \mathbb{N}}$

Let $(x, y) \in \mathbb{R}^{2}$. By the slope constraint for functions in $\Gamma$, it is easy to check that for any $n \in \mathbb{N}, x \in \mathbb{R}$ and $y<y^{\prime}$ :

$$
S_{n}(s, t, f ; \omega)\left(x, y^{\prime}\right)-S_{n}(s, t, f ; \omega)(x, y) \in\left[-\left(\left\lfloor n y^{\prime}\right\rfloor-\lfloor n y\rfloor\right) / n, 0\right] .
$$

By this and Proposition 5.6,

$$
\begin{aligned}
\limsup _{n \rightarrow \infty} & \sup _{\substack{\left(x^{\prime}, y^{\prime}\right) \in \mathbb{R}^{2} \\
\left|x-x^{\prime}\right|,\left|y-y^{\prime}\right| \leq \delta}}\left|S_{n}(s, t, f ; \omega)(x, y)-S_{n}(s, t, f ; \omega)\left(x^{\prime}, y^{\prime}\right)\right| \\
& \leq \limsup _{n \rightarrow \infty} \sup _{x^{\prime} \in[x-\delta, x+\delta]}\left|S_{n}(s, t, f ; \omega)(x, y)-S_{n}(s, t, f ; \omega)\left(x^{\prime}, y\right)\right|+\delta \\
& \leq \sup _{\substack{x_{1}, x_{2} \in[x-\delta-T, x+\delta+T] \\
\left|x_{2}-x_{1}\right| \leq 2 \delta}}\left|f\left(x_{1}, y\right)-f\left(x_{2}, y\right)\right|+C \sqrt{\delta}+\delta .
\end{aligned}
$$

By uniform continuity of $f$ on any compact, the right-hand side tends to 0 when $\delta$ tends to 0 .

Therefore, by Proposition D.1, any subsequence of $\left(S_{n}(s, t, f ; \omega)\right)_{n \in \mathbb{N}}$ has a subsequence that converges in $\left(\mathcal{F}\left(\mathbb{R}^{2}\right), d_{\infty, c}\right)$ and any limit point is continuous. Actually, by taking the limit in (5.10), any limit point is in $\bar{\Gamma}$. This concludes the proof of Lemma 5.13.

To finish the proof of Lemma 5.12, we are going to show asymptotic equi-continuity of $\left((s, t) \mapsto S_{n}(s, t, f ; \omega)\right)_{n \in \mathbb{N}}$. Let us fix $\omega \in \Omega_{0}, f \in \bar{\Gamma}$ and $(s, t) \in \mathcal{T}$. By definition of $d_{\infty, c}$, it is enough to show that for any $\varepsilon>0$ and $R>0$ there exists $\delta>0$ such that:

$$
\limsup _{n \rightarrow \infty} \sup _{\substack{\left(s^{\prime}, t^{\prime}\right) \in \mathcal{T} \\\left|s^{\prime}-s\right| \leq \delta,\left|t^{\prime}-t\right| \leq \delta}}\left\|S_{n}(s, t, f ; \omega)-S_{n}\left(s^{\prime}, t^{\prime}, f ; \omega\right)\right\|_{\infty}^{[-R, R]^{2}} \leq \varepsilon .
$$

We claim that for any $\left(s^{\prime}, t^{\prime}\right) \in \mathcal{T}$, there exists some $r \leq t \wedge t^{\prime}$ and $u \geq s \vee s^{\prime}$ such that

$$
\begin{aligned}
& \left\|S_{n}(s, t, f ; \omega)-S_{n}\left(s^{\prime}, t^{\prime}, f ; \omega\right)\right\|_{\infty}^{[-R, R]^{2}} \\
& \leq\left\|S_{n}(r, t, f ; \omega)-S_{n}\left(r, t^{\prime}, f ; \omega\right)\right\|_{\infty}^{[-R, R]^{2}}+\left\|S_{n}(s, u, f ; \omega)-S_{n}\left(s^{\prime}, u, f ; \omega\right)\right\|_{\infty}^{[-R, R]^{2}} .
\end{aligned}
$$

Indeed, at least one of the two conditions occurs: $s \leq t^{\prime}$ or $s^{\prime} \leq t$. In the first case, (5.12) holds with $(r, u)=\left(s, t^{\prime}\right)$ while in the second case, $(r, u)=\left(s^{\prime}, t\right)$. Therefore, asymptotic equi-continuity of $\left((s, t) \mapsto S_{n}(s, t, f ; \omega)\right)_{n \in \mathbb{N}}$ follows from the next Lemma.

Lemma 5.14. For all $\omega \in \Omega_{0},(s, t) \in \mathcal{T}, f \in \bar{\Gamma}, R>0$ and $\varepsilon>0$, there exists $\delta>0$ such that

$$
\limsup _{n \rightarrow \infty} \sup _{\substack{r, t^{\prime} \in[0, T] \\ r \leq t \wedge t^{\prime},\left|t^{\prime}-t\right| \leq \delta}}\left\|S_{n}(r, t, f ; \omega)-S_{n}\left(r, t^{\prime}, f ; \omega\right)\right\|_{\infty}^{[-R, R]^{2}} \leq \varepsilon
$$

and

$$
\limsup _{n \rightarrow \infty} \sup _{\substack{u, s^{\prime} \in[0, T] \\ u \geq s \vee s^{\prime},\left|s^{\prime}-s\right| \leq \delta}}\left\|S_{n}(s, u, f ; \omega)-S_{n}\left(s^{\prime}, u, f ; \omega\right)\right\|_{\infty}^{[-R, R]^{2}} \leq \varepsilon .
$$


Proof. We are going to prove (5.13) first. Let $(x, y) \in \mathbb{R}^{2}$ and suppose first that $r \leq t \leq t^{\prime}$. By Lemma 4.4,

$$
\begin{aligned}
S_{n}\left(r, t^{\prime}, f ; \omega\right)(x, y) & =\frac{1}{n} h\left(n x,\lfloor n y\rfloor, n\left(t^{\prime}-r\right), \varphi_{n}^{f} ; \tau_{n r} \omega\right) \\
& =\frac{1}{n} h\left(n x,\lfloor n y\rfloor, n\left(t^{\prime}-t\right), h\left(n \cdot,\lfloor n \cdot\rfloor, n(t-r), \varphi_{n}^{f}, \tau_{n r} \omega\right) ; \tau_{n t} \omega\right) .
\end{aligned}
$$

Now, by Lemma 5.3 applied with initial condition $\psi:=h\left(n \cdot,\lfloor n \cdot\rfloor, n(t-r), \varphi_{n}^{f}, \tau_{n r} \omega\right)$,

$$
\begin{aligned}
S_{n}\left(r, t^{\prime}, f ; \omega\right)(x, y) & =\sup _{|z-x| \leq\left|t^{\prime}-t\right|}\left\{\frac{1}{n} \psi(z, y)+\frac{1}{n} L^{\uparrow}\left(\left(\tau_{n t} \omega\right)_{\lfloor n y\rfloor}^{\psi} \cap n R_{(z, 0),\left(x, t^{\prime}-t\right)}\right)\right\} \\
& \leq \sup _{|z-x| \leq\left|t^{\prime}-t\right|}\left\{\frac{1}{n} \psi(z, y)+\frac{1}{n} L^{\uparrow}\left(\left(\tau_{n t} \omega\right)_{\lfloor n y\rfloor} \cap n R_{(z, 0),\left(x, t^{\prime}-t\right)}\right)\right\} \\
& =\sup _{|z-x| \leq\left|t^{\prime}-t\right|}\left\{S_{n}(r, t, f ; \omega)(z, y)+\frac{1}{n} L^{\uparrow}\left(\omega_{\lfloor n y\rfloor} \cap n R_{(z, t),\left(x, t^{\prime}\right)}\right)\right\} \\
& \leq \sup _{|z-x| \leq\left|t^{\prime}-t\right|} S_{n}(r, t, f ; \omega)(z, y)+\frac{1}{n} L^{\uparrow}\left(\omega_{\lfloor n y\rfloor} \cap n R_{\left(x, t-\left|t^{\prime}-t\right|\right),\left(x, t^{\prime}\right)}\right),
\end{aligned}
$$

since $R_{(z, t),\left(x, t^{\prime}\right)} \subseteq R_{\left(x, t-\left|t^{\prime}-t\right|\right),\left(x, t^{\prime}\right)}$ for all $|z-x| \leq\left|t^{\prime}-t\right|$. Similarly if $r \leq t^{\prime} \leq t$,

$$
S_{n}(r, t, f ; \omega)(x, y) \leq \sup _{|z-x| \leq\left|t^{\prime}-t\right|} S_{n}\left(r, t^{\prime}, f ; \omega\right)(z, y)+\frac{1}{n} L^{\uparrow}\left(\omega_{\lfloor n y\rfloor} \cap n R_{\left(x, t^{\prime}-\left|t^{\prime}-t\right|\right),(x, t)}\right) .
$$

In any case, since $R_{(x, t-2 \delta),(x, t+\delta)}$ contains both $R_{\left(x, t-\left|t^{\prime}-t\right|\right),\left(x, t^{\prime}\right)}$ and $R_{\left(x, t^{\prime}-\left|t^{\prime}-t\right|\right),(x, t)}$,

$$
\begin{aligned}
& \sup _{\substack{r, t^{\prime} \in[0, T] \\
r \leq t \wedge t^{\prime},\left|t^{\prime}-t\right| \leq \delta}}\left|S_{n}\left(r, t^{\prime}, f ; \omega\right)(x, y)-S_{n}(r, t, f ; \omega)(x, y)\right| \\
& \leq \sup _{\substack{z \in[x-\delta, x+\delta] \\
r \leq t \wedge t^{\prime}}}\left|S_{n}\left(r, t \wedge t^{\prime}, f ; \omega\right)(z, y)-S_{n}\left(r, t \wedge t^{\prime}, f ; \omega\right)(x, y)\right| \\
& \quad+\frac{1}{n} L^{\uparrow}\left(\omega_{\lfloor n y]} \cap n R_{(x, t-2 \delta),(x, t+\delta)}\right) .
\end{aligned}
$$

Therefore, by Proposition 5.6 and Lemma 5.4, since Leb $\left(R_{(x, t-2 \delta),(x, t+\delta)}\right)=(3 \delta)^{2} / 2$,

$$
\begin{aligned}
\limsup _{n \rightarrow \infty} \sup _{\substack{r, t^{\prime} \in[0, T] \\
r \leq t \wedge t^{\prime},\left|t^{\prime}-t\right| \leq \delta}}\left|S_{n}\left(r, t^{\prime}, f ; \omega\right)(x, y)-S_{n}(r, t, f ; \omega)(x, y)\right| \\
\quad \leq \sup _{\substack{x_{1}, x_{2} \in[x-\delta-T, x+\delta+T] \\
\left|x_{2}-x_{1}\right| \leq 2 \delta}}\left|f\left(x_{1}, y\right)-f\left(x_{2}, y\right)\right|+C \sqrt{\delta}+c \frac{3}{\sqrt{2}} \delta .
\end{aligned}
$$

The right-hand side tends to 0 when $\delta$ goes to 0 . To finish off the proof of (5.13), we need to get a uniform control in $(x, y) \in[-R, R]^{2}$. To do this, we cover the rectangle $[-R, R]^{2}$ by a finite union of balls of radius $\delta$. Let $\left(x_{1}, y_{1}\right), \cdots,\left(x_{p}, y_{p}\right)$ be the centers of these balls. By (5.11), for any $i$,

$$
\begin{aligned}
\limsup _{n \rightarrow \infty} \sup _{\substack{\left(r, r^{\prime}\right) \in \mathcal{T} \\
(x, y) \in \mathcal{B}\left(\left(x_{i}, y_{i}\right), \delta\right)}}\left|S_{n}\left(r, r^{\prime}, f ; \omega\right)(x, y)-S_{n}\left(r, r^{\prime}, f ; \omega\right)\left(x_{i}, y_{i}\right)\right| \\
\leq \sup _{x_{1}, x_{2} \in\left[x_{-}-\delta-T, x+\delta+T\right]}\left|f\left(x_{1}, y\right)-f\left(x_{2}, y\right)\right|+C \sqrt{\delta}+\delta .
\end{aligned}
$$

This bound proves the uniform control in $(x, y) \in \mathcal{B}\left(\left(x_{i}, y_{i}\right), \delta\right)$. Since (5.15) holds simultaneously for all $\left(x_{i}, y_{i}\right)$, (5.13) holds for any $\delta>0$ chosen small enough. 
Let us now prove (5.14). If $s \leq s^{\prime} \leq u$, by Lemma 4.4,

$$
\begin{aligned}
S_{n}(s, u, f ; \omega) & =\frac{1}{n} h\left(n \cdot,\lfloor n \cdot\rfloor, n\left(u-s^{\prime}\right), h\left(n \cdot,\lfloor n \cdot\rfloor, n\left(s^{\prime}-s\right), \varphi_{n}^{f} ; \tau_{n s} \omega\right) ; \tau_{n s^{\prime}} \omega\right), \\
\text { and } \quad S_{n}\left(s^{\prime}, u, f ; \omega\right) & =\frac{1}{n} h\left(n \cdot,\lfloor n \cdot\rfloor, n\left(u-s^{\prime}\right), \varphi_{n}^{f} ; \tau_{n s^{\prime}} \omega\right) .
\end{aligned}
$$

Therefore, by Corollary 4.8, there exists $N(\omega) \in \mathbb{N}$ such that for all $n \geq N(\omega)$ and all $s \leq s^{\prime} \leq u \leq T$,

$$
\begin{aligned}
\| S_{n}(s, u, f ; \omega) & -S_{n}\left(s^{\prime}, u, f ; \omega\right) \|_{\infty}^{[-R, R]^{2}} \\
& \leq\left\|\frac{1}{n} h\left(n \cdot,\lfloor n \cdot\rfloor, n\left(s^{\prime}-s\right), \varphi_{n}^{f} ; \tau_{n s} \omega\right)-\frac{1}{n} \varphi_{n}^{f}(n \cdot,\lfloor n \cdot])\right\|_{\infty}^{\left[-R-\alpha\left(u-s^{\prime}\right), R+\alpha\left(u-s^{\prime}\right)\right]^{2}} \\
& \leq\left\|S_{n}\left(s, s^{\prime}, f ; \omega\right)-S_{n}(s, s, f ; \omega)\right\|_{\infty}^{[-R-\alpha T, R+\alpha T]^{2}} .
\end{aligned}
$$

We can do similarly for $s^{\prime} \leq s \leq u$ and finally get that for all $n \geq N(\omega)$,

$$
\begin{aligned}
\sup _{\substack{u, s^{\prime} \in[0, T] \\
u \geq s \vee s^{\prime},\left|s^{\prime}-s\right| \leq \delta}}\left\|S_{n}(s, u, f ; \omega)-S_{n}\left(s^{\prime}, u, f ; \omega\right)\right\|_{\infty}^{[-R, R]^{2}} \\
\quad \leq \sup _{\substack{s^{\prime} \in[0, T] \\
\left|s-s^{\prime}\right| \leq \delta}}\left\|S_{n}\left(s \wedge s^{\prime}, s, f ; \omega\right)-S_{n}\left(s \wedge s^{\prime}, s^{\prime}, f ; \omega\right)\right\|_{\infty}^{[-R-\alpha T, R+\alpha T]^{2}}
\end{aligned}
$$

and the proof is concluded by the first case (5.13) (with $\left(t, t^{\prime}\right)=\left(s, s^{\prime}\right)$ and $r=s \wedge s^{\prime}$ ).

This shows the asymptotic equi-continuity of $\left((s, t) \mapsto S_{n}(s, t, f ; \omega)\right)_{n \in \mathbb{N}}$. Together with Lemma 5.13 and Proposition D.1, this concludes the proof of Lemma 5.12.

The proof of Proposition 5.10 is complete up to showing Lemma 5.11.

Proof of Lemma 5.11. Let $\omega \in \Omega_{0}, \varepsilon>0$ and $I \in \mathbb{N}$ such that $2^{-I} \leq \varepsilon / 2$. By definition of the metric $D_{\infty}$, for any $f, g \in \bar{\Gamma}$,

$$
\begin{array}{r}
D_{\infty}\left(S_{n}(\cdot, \cdot, f ; \omega), S_{n}(\cdot, \cdot, g ; \omega)\right)=\sup _{0 \leq s \leq t \leq T} \sum_{i=1}^{\infty} 2^{-i}\left(\left\|S_{n}(s, t, f ; \omega)-S_{n}(s, t, g ; \omega)\right\|_{\infty}^{[-i, i]^{2}} \wedge 1\right) \\
\leq \sup _{0 \leq s \leq t \leq T} \sum_{i=1}^{I} 2^{-i}\left(\left\|S_{n}(s, t, f ; \omega)-S_{n}(s, t, g ; \omega)\right\|_{\infty}^{[-i, i]^{2}} \wedge 1\right)+\varepsilon / 2 .
\end{array}
$$

Now, by (4.12), there exists $N(\omega) \in \mathbb{N}$ such that for all $n \geq N(\omega)$ for all $f, g \in \bar{\Gamma}$ and all $0 \leq s \leq t \leq T$,

$$
\begin{aligned}
\left\|S_{n}(s, t, f ; \omega)-S_{n}(s, t, g ; \omega)\right\|_{\infty}^{[-i, i]^{2}} & \leq\left\|\frac{1}{n} \varphi_{n}^{f}(n \cdot,\lfloor n \cdot\rfloor)-\frac{1}{n} \varphi_{n}^{g}(n \cdot,\lfloor n \cdot\rfloor)\right\|_{\infty}^{[-i-\alpha T, i+\alpha T]^{2}} \\
& \leq\|f-g\|_{\infty}^{[-i-\alpha T, i+\alpha T]^{2}}+4 / n . \quad \text { by (4.15) }
\end{aligned}
$$

Therefore, for all $n \geq \max (N(\omega), \varepsilon / 8)$ and all $f, g \in \bar{\Gamma}$,

$$
\begin{aligned}
D_{\infty}\left(S_{n}(\cdot, \cdot, f ; \omega), S_{n}(\cdot, \cdot, g ; \omega)\right) & \leq \sum_{i=1}^{I} 2^{-i}\left(\|f-g\|_{\infty}^{[-i-\alpha T, i+\alpha T]^{2}} \wedge 1\right)+4 / n+\varepsilon / 2 \\
& \leq 2^{\lceil\alpha T\rceil} \sum_{i=1}^{\infty} 2^{-i-\lceil\alpha T\rceil}\left(\|f-g\|_{\infty}^{[-i-\lceil\alpha T\rceil, i+\lceil\alpha T\rceil]^{2}} \wedge 1\right)+\varepsilon \\
& \leq 2^{\lceil\alpha T\rceil} d_{\infty, c}(f, g)+\varepsilon .
\end{aligned}
$$




\section{Identification of the limit}

\subsection{Properties of the limit points}

In this section, we are going to show that any subsequential limit of $\left(S_{n}(.,, ; f, \omega)\right)_{n \in \mathbb{N}}$ (as in Definition 4.9) satisfies the sufficient conditions of Proposition 3.5, most of these properties being automatically satisfied by the analogous microscopic properties stated in Section 4.1 or by Proposition 5.10 concerning continuity.

Proposition 6.1. Let $\omega \in \Omega_{0}$ and $\left(n_{k}\right)_{k \in \mathbb{N}}$ a subsequence such that for all $f \in \bar{\Gamma}$, $\left(S_{n_{k}}(\cdot, \cdot ; f, \omega)\right)_{k \in \mathbb{N}}$ converges to a certain $S(\cdot, \cdot ; f, \omega)$ in $\mathcal{F}_{\mathcal{T}}$, i.e

$$
\forall R>0, \quad \sup _{\substack{(s, t) \in \mathcal{T} \\|x|,|y| \leq R}}\left|S_{n_{k}}(s, t, f ; \omega)(x, y)-S(s, t, f ; \omega)(x, y)\right| \underset{k \rightarrow \infty}{\longrightarrow} 0 .
$$

Any such limit $(f \mapsto S(s, t, f ; \omega))_{0 \leq s \leq t \leq T}$ is a family of continuous functions from $\bar{\Gamma}$ into itself satisfying the first four properties listed in Proposition 3.5. Moreover, for any $f \in \bar{\Gamma}$, $(s, t, x, y) \mapsto S(s, t, f ; \omega)(x, y)$ is continuous.

Proof. - Continuity: By Proposition 5.10, for all $f \in \bar{\Gamma},(s, t) \mapsto S(s, t, f ; \omega)$ is continuous from $\mathcal{T}$ into $\bar{\Gamma}$ (which is composed of continuous functions) hence $(s, t, x, y) \mapsto$ $S(s, t, f ;, \omega)(x, y)$ is continuous.

- Translation invariance: For any $c \in \mathbb{R}, s \leq t$ and $k \in \mathbb{N}$, by Lemma 4.2 and by translation invariance property of $\varphi_{n}^{f}$ stated in Proposition 4.10,

$$
S_{n_{k}}\left(s, t ; f+n_{k}^{-1}\left\lfloor n_{k} c\right\rfloor, \omega\right)=S_{n_{k}}(s, t ; f, \omega)+\frac{1}{n_{k}}\left\lfloor n_{k} c\right\rfloor .
$$

When $k$ goes to infinity, the right-hand side tends to $S(s, t ; f, \omega)+c$ in $\left(\mathcal{F}\left(\mathbb{R}^{2}\right), d_{\infty, c}\right)$ while the left-hand side goes to $S(s, t ; f+c, \omega)$ by Lemma 5.11 .

- Monotonicity: By (4.15), if $f \leq g$, then for all $k \in \mathbb{N}, \varphi_{n_{k}}^{f} \leq \varphi_{n_{k}}^{g}+4$ so by Lemmas 4.3 and 4.2,

$$
S_{n_{k}}(s, t, f ; \omega) \leq S_{n_{k}}(s, t, g ; \omega)+4 / n_{k} .
$$

Monotonicty follows by taking the limit $k \rightarrow \infty$.

- Locality: It is a direct consequence of Corollary 4.8 and (4.15).

- Semi-group: the fact that $S(t, t, f)=f$, for all $t \in[0, T]$ and $f \in \bar{\Gamma}$ is an immediate consequence of (4.15). Now, for any $0 \leq r \leq s \leq t \leq T$, we have by Lemma 4.4,

$$
S_{n_{k}}(r, t, f ; \omega)=\frac{1}{n_{k}} h\left(n \cdot\lfloor n \cdot\rfloor, n(t-s), h\left(\cdot, \cdot, n(s-r), \varphi_{n_{k}}^{f} ; \tau_{n r} \omega\right) ; \tau_{n s} \omega\right),
$$

and since $S(r, s, f ; \omega) \in \bar{\Gamma}$, we can apply $S_{n_{k}}(s, t, \cdot ; \omega)$ and write

$$
S_{n_{k}}(s, t, S(r, s, f ; \omega) ; \omega)=\frac{1}{n_{k}} h\left(n \cdot,\lfloor n \cdot\rfloor, n(t-s), \varphi_{n_{k}}^{S(r, s, f ; \omega)} ; \tau_{n s} \omega\right) .
$$

Therefore, by Corollary 4.8, for all $R \geq 0$ and $k$ large enough,

$$
\begin{aligned}
\| S_{n_{k}}(r, t, f ; \omega) & -S_{n_{k}}(s, t, S(r, s, f ; \omega) ; \omega) \|_{\infty}^{[-R, R]^{2}} \\
\leq & \left\|\frac{1}{n_{k}} h\left(n \cdot,\lfloor n \cdot\rfloor, n(s-r), \varphi_{n_{k}}^{f} ; \tau_{n r} \omega\right)-\frac{1}{n_{k}} \varphi_{n_{k}}^{S(r, s, f ; \omega)}(n \cdot,\lfloor n \cdot\rfloor)\right\|_{\infty}^{[-R-\alpha T, R+\alpha T]^{2}} \\
& \leq\left\|S_{n_{k}}(r, s, f ; \omega)-S(r, s, f ; \omega)\right\|_{\infty}^{[-R-\alpha T, R+\alpha T]^{2}}+\frac{2}{n_{k}} \quad \text { by (4.15) }
\end{aligned}
$$

which tends to zero when $k$ goes to infinity. Consequently, for all $R \geq 0$,

$$
\begin{aligned}
\| S(r, t, f ; \omega)-S(s, t & , S(r, s, f ; \omega) ; \omega) \|_{\infty}^{[-R, R]^{2}} \\
= & \lim _{k \rightarrow \infty}\left\|S_{n_{k}}(r, t, f ; \omega)-S_{n_{k}}(s, t, S(r, s, f ; \omega) ; \omega)\right\|_{\infty}^{[-R, R]^{2}}=0,
\end{aligned}
$$

which concludes the proof of the semi-group property. 


\subsection{Hydrodynamic limit for linear initial profiles}

The only condition missing to apply Proposition 3.5 is the compatibility with linear initial profiles. We start with the following result:

Proposition 6.2. For all $\rho \in \mathbb{R} \times(-1,0)$, all $t \in[0, T]$ and all $(x, y) \in \mathbb{R}^{2}$ :

$$
\omega-\text { a.s } \quad S_{n}\left(0, t ; f_{\rho}, \omega\right)(x, y) \underset{n \rightarrow \infty}{\longrightarrow} f_{\rho}(x, y)+t v(\rho)
$$

with $f_{\rho}:=(x, y) \mapsto \rho \cdot(x, y)$.

Before proving this Proposition, let us show the following Corollary that gives the compatibility with linear solutions.

Corollary 6.3. There exists $\Omega_{1} \subseteq \Omega$ of probability one such that for all $\omega \in \Omega_{0} \cap \Omega_{1}$, if $\left(n_{k}\right)_{k \in \mathbb{N}}$ is a subsequence such that for all $f \in \bar{\Gamma},\left(S_{n_{k}}(\cdot, \cdot ; f, \omega)\right)_{k \in \mathbb{N}}$ converges towards $S(\cdot, \cdot ; f, \omega)$ in $\mathcal{F}_{\mathcal{T}}$, then

$$
\forall \rho \in \mathbb{R} \times[-1,0] \quad \forall 0 \leq s \leq t \leq T \quad S\left(s, t, f_{\rho} ; \omega\right)=f_{\rho}+(t-s) v(\rho) .
$$

Proof of Corollary 6.3. By Proposition 6.2, there exists a subset $\Omega_{1} \subseteq \Omega$ of probability one such that (6.1) holds for any $\rho, t, x, y$ in a countable dense subset of their respective set of definition. Therefore, for all $\omega \in \Omega_{0} \cap \Omega_{1}$, any subsequential limit $S(\cdot, \cdot, \cdot ; \omega)$ of $S_{n}(\cdot, \cdot, \cdot ; \omega)$ satisfies that for any such $\rho, t, x, y$,

$$
S\left(0, t, f_{\rho} ; \omega\right)(x, y)=f_{\rho}(x, y)+t v(\rho)
$$

By continuity with respect to $(t, x, y)$ of both sides (by Proposition 6.1), this holds actually for all $(x, y) \in \mathbb{R}^{2}$ and $t \in[0, T]$. Similarly, by continuity of $\rho \mapsto v(\rho)$ (defined in (3.6)) and of $\rho \mapsto f_{\rho}$ on $\mathbb{R} \times[-1,0]$ (including the endpoints of the interval) for the topology of convergence on all compact sets and by continuity of $f \mapsto S(0, t, f ; \omega)$ for the same topology (still by Proposition 6.1) we deduce that it holds also for all $\rho \in \mathbb{R} \times[-1,0]$. Finally, we get the result for any $s>0$ by the semi-group property satisfied by $S$ (by Proposition 6.1):

$$
\begin{aligned}
f_{\rho}+t v(\rho)=S\left(0, t, f_{\rho} ; \omega\right) & =S\left(s, t, S\left(0, s, f_{\rho}, \omega\right) ; \omega\right) \\
\quad=S\left(s, t, f_{\rho}+s v(\rho) ; \omega\right) & =S\left(s, t, g_{\rho} ; \omega\right)+s v(\rho) \quad \text { by translation invariance property }
\end{aligned}
$$

and thus $S\left(s, t, f_{\rho} ; \omega\right)=f_{\rho}+(t-s) v(\rho)$.

Proof of Proposition 6.2. This proof requires the knowledge on equilibrium measures developed by Prähofer and Spohn in $[20,19]$. As in Section 3.3, we note $\varphi_{M, N, \rho}$ the height function with asymptotic average slope $\rho \in \mathbb{R} \times(-1,0)$ (in the thermodynamic limit $N \rightarrow \infty, M \rightarrow \infty$ ) and whose gradients are stationary w.r.t time for the periodised Gates-Westcott dynamic (i.e the Poisson point process is periodised on a torus of size $2 M$ and $2 N$ and noted $[\omega]^{M, N}$ as in (3.9)). There are two key ingredients in this proof: to show that, in the limit $M, N \rightarrow \infty, \varphi_{M, N, \rho}$ approaches $f_{\rho}$ in the sense of (3.3) and that $n^{-1} h\left(0,0, n t ; \varphi_{M, N, \rho},[\omega]^{M, N}\right)$ approaches $f_{\rho}+t v(\rho)$. From (3.10) and (3.12), this is true on average. It remains to show concentration via variance estimates as in the next Lemmas.

Lemma 6.4. For any $\rho \in \mathbb{R} \times(-1,0)$ and $t \geq 0$,

$$
\limsup _{N \rightarrow \infty} \limsup _{M \rightarrow \infty} \operatorname{Var}\left(h\left(0,0, t ; \varphi_{M, N, \rho},[\omega]^{M, N}\right)\right)=\underset{t \rightarrow \infty}{\mathrm{O}}(\log t) .
$$

Lemma 6.5. For any $\rho \in \mathbb{R} \times(-1,0)$, any $\varepsilon>0$, any $n \in \mathbb{N}^{*}$ and any compact set $K \subseteq \mathbb{R}^{2}$,

$$
\limsup _{N \rightarrow \infty} \limsup _{M \rightarrow \infty} \mathbb{P}\left(\sup _{(x, y) \in K}\left|\frac{1}{n} \varphi_{M, N, \rho}(n x,\lfloor n y\rfloor)-f_{\rho}(x, y)\right| \geq \varepsilon\right)=\underset{n \rightarrow \infty}{\mathrm{O}}\left(n^{-2} \log n\right) .
$$


Let us admit first these Lemmas and finish the proof of Proposition 6.2. Let us fix $\rho, t, x, y$ as in Proposition 6.2. We also fix $\varepsilon>0$ and $n \in \mathbb{N}$. For any $M, N \in \mathbb{R}_{+}$,

$$
\begin{array}{rlr}
\mathbb{P} & \left.|| S_{n}\left(0, t ; f_{\rho}, \omega\right)(x, y)-f_{\rho}(x, y)-t v(\rho) \mid \geq 2 \varepsilon\right) & \\
& \leq \mathbb{P}\left(S_{n}\left(0, t ; f_{\rho}, \omega\right)(x, y) \neq S_{n}\left(0, t ; f_{\rho},[\omega]^{M, N}\right)(x, y)\right) & \} \mathrm{B} \\
\quad+\mathbb{P}\left(\left|n^{-1} h\left(n x,\lfloor n y\rfloor, n t ; \varphi_{M, N, \rho},[\omega]^{M, N}\right)-f_{\rho}(x, y)-t v(\rho)\right| \geq \varepsilon\right) & \\
\quad+\mathbb{P}\left(\left|S_{n}\left(0, t ; f_{\rho},[\omega]^{M, N}\right)(x, y)-n^{-1} h\left(n x,\lfloor n y\rfloor, n t ; \varphi_{M, N, \rho},[\omega]^{M, N}\right)\right| \geq \varepsilon\right) . & \} \mathrm{C}
\end{array}
$$

Let us bound the limsup when $M, N$ goes to infinity of the three terms of the r.h.s called A, B and C.

A) The first term is easy to control thanks to the linear propagation of information. For any $M, N$ large enough, $[-M, M) \times \llbracket-N, N-1 \rrbracket$ contains $\mathcal{B}\left((n x, n y), \alpha_{M, N} n t\right)$ with $\alpha_{M, N}:=(M \wedge N) /(2 n t)$. For such $M, N$, if $\omega \in A_{n(x, y), 0, n t, \alpha_{M, N}}$ (defined in (4.9)), then $S_{n}\left(0, t ; f_{\rho}, \omega\right)(x, y)=S_{n}\left(0, t ; f_{\rho},[\omega]^{M, N}\right)(x, y)$. Consequently, by (4.10) and since $\alpha_{M, N}$ tends to infinity when $M, N$ tend to infinity,

$$
\mathbb{P}\left(S_{n}\left(0, t ; f_{\rho}, \omega\right)(x, y) \neq S_{n}\left(0, t ; f_{\rho},[\omega]^{M, N}\right)(x, y)\right) \leq \mathbb{P}\left({ }^{c} A_{n(x, y), 0, n t, \alpha_{M, N}}\right) \underset{M, N \rightarrow \infty}{\longrightarrow} 0 .
$$

B) Let us write $h\left(n x,\lfloor n y\rfloor, n t ; \varphi_{M, N, \rho}\right)$ for $h\left(n x,\lfloor n y\rfloor, n t ; \varphi_{M, N, \rho},[\omega]^{M, N}\right)$. By Chebyshev's inequality,

$$
\begin{aligned}
& \mathbb{P}\left(\left|n^{-1} h\left(n x,\lfloor n y\rfloor, n t ; \varphi_{M, N, \rho}\right)-f_{\rho}(x, y)-t v(\rho)\right| \geq \varepsilon\right) \\
& \leq \varepsilon^{-2} \mathbb{E}\left[\left|n^{-1} h\left(n x,\lfloor n y\rfloor, n t ; \varphi_{M, N, \rho}\right)-f_{\rho}(x, y)-t v(\rho)\right|^{2}\right] \\
& =\varepsilon^{-2}\left(\mathbb{E}\left[n^{-1} h\left(n x,\lfloor n y\rfloor, n t ; \varphi_{M, N, \rho}\right)\right]-f_{\rho}(x, y)-t v(\rho)\right)^{2} \\
& \quad+\varepsilon^{-2} \frac{\operatorname{Var}\left(h\left(n x,\lfloor n y\rfloor, n t ; \varphi_{M, N, \rho}\right)\right)}{n^{2}} .
\end{aligned}
$$

By (3.12), the first term of the r.h.s in the last equality goes to zero when $M, N$ tends to infinity. To treat the second term, we write $h\left(n x,\lfloor n y\rfloor, n t ; \varphi_{M, N, \rho}\right)=h\left(0,0, n t ; \varphi_{M, N, \rho}\right)+$ $h\left(n x,\lfloor n y\rfloor, n t ; \varphi_{M, N, \rho}\right)-h\left(0,0, n t ; \varphi_{M, N, \rho}\right)$ and use that the variance of the sum is smaller than twice the sum of the variances:

$$
\begin{aligned}
\operatorname{Var} & \left(h\left(n x,\lfloor n y\rfloor, n t ; \varphi_{M, N, \rho}\right)\right) \\
& \leq 2 \operatorname{Var}\left(h\left(0,0, n t ; \varphi_{M, N, \rho}\right)\right)+2 \operatorname{Var}\left(h\left(n x,\lfloor n y\rfloor, n t ; \varphi_{M, N, \rho}\right)-h\left(0,0, n t ; \varphi_{M, N, \rho}\right)\right) \\
& =2 \operatorname{Var}\left(h\left(0,0, n t ; \varphi_{M, N, \rho}\right)\right)+2 \operatorname{Var}\left(\varphi_{M, N, \rho}(n x,\lfloor n y\rfloor)\right), \quad \text { by (3.11). }
\end{aligned}
$$

The first term of the r.h.s is controlled by Lemma 6.4 and the second by (3.13). Therefore,

$$
\limsup _{N \rightarrow \infty} \limsup _{M \rightarrow \infty} \mathbb{P}\left(\left|n^{-1} h\left(n x,\lfloor n y\rfloor, n t ; \varphi_{M, N, \rho}\right)-f_{\rho}(x, y)-t v(\rho)\right| \geq \varepsilon\right)=\underset{n \rightarrow \infty}{\mathrm{O}}\left(n^{-2} \log n\right) .
$$

C) By Lemma 4.7 and by (4.15), for any $n \geq 2 / \varepsilon$

$$
\begin{aligned}
& \mathbb{P}\left(\left|S_{n}\left(0, t ; f_{\rho},[\omega]^{M, N}\right)(x, y)-n^{-1} h\left(n x,\lfloor n y\rfloor, n t ; \varphi_{M, N, \rho},[\omega]^{M, N}\right)\right| \geq \varepsilon\right) \\
& \quad \leq \mathbb{P}\left(\sup _{\left(x^{\prime}, y^{\prime}\right) \in \mathcal{B}((x, y), \alpha t)}\left|f_{\rho}\left(x^{\prime}, y^{\prime}\right)-\frac{1}{n} \varphi_{M, N, \rho}\left(n x^{\prime},\left\lfloor n y^{\prime}\right\rfloor\right)\right| \geq \varepsilon / 2\right)+\operatorname{Or\infty }_{n \rightarrow \infty}\left(e^{-\gamma n}\right) .
\end{aligned}
$$

Consequently, by Lemma 6.5,

$$
\begin{array}{r}
\limsup _{N \rightarrow \infty} \limsup _{M \rightarrow \infty} \mathbb{P}\left(\left|S_{n}\left(0, t ; f_{\rho},[\omega]^{M, N}\right)(x, y)-n^{-1} h\left(n x,\lfloor n y\rfloor, n t ; \varphi_{M, N, \rho},[\omega]^{M, N}\right)\right| \geq \varepsilon\right) \\
=\underset{n \rightarrow \infty}{\mathrm{O}}\left(n^{-2} \log n\right) .
\end{array}
$$


Altogether, by taking the limsup when $M, N$ goes to infinity in (6.5) and by (6.6), (6.7) and (6.8),

$$
\mathbb{P}\left(\left|S_{n}\left(0, t ; f_{\rho}, \omega\right)(x, y)-f_{\rho}(x, y)-t v(\rho)\right| \geq 2 \varepsilon\right)=\underset{n \rightarrow \infty}{\mathrm{O}}\left(n^{-2} \log n\right),
$$

and the proof of Proposition 6.2 follows from Borel-Cantelli Lemma.

Now, as promised, we prove Lemmas 6.4 and 6.5.

Proof of Lemma 6.4. Again we write $h\left(x, y, t ; \varphi_{M, N, \rho}\right)$ instead of $h\left(x, y, t ; \varphi_{M, N, \rho},[\omega]^{M, N}\right)$. For any rectangle $\Lambda_{R}=[-R, R] \times \llbracket-R, R \rrbracket$ with $R>0$ and any $t \geq 0$ if we define

$$
h\left(\Lambda_{R}, t ; \varphi_{M, N, \rho}\right):=\sum_{y=-R}^{R} \int_{-R}^{R} h\left(x, y, t ; \varphi_{M, N, \rho}\right) \mathrm{d} x,
$$

then it is easy to see

$$
h\left(\Lambda_{R}, t ; \varphi_{M, N, \rho}\right)-h\left(\Lambda_{R}, 0 ; \varphi_{M, N, \rho}\right)=\int_{0}^{t}\left(N_{M, N, \rho}^{+}\left(\Lambda_{R}, s\right)+N_{M, N, \rho}^{-}\left(\Lambda_{R}, s\right)\right) \mathrm{d} s,
$$

where $N_{M, N, \rho}^{ \pm}\left(\Lambda_{R}, s\right)$ is the number of antikinks/kinks in the domain $\Lambda_{R}$ at time $s$ for the dynamic starting from $\varphi_{M, N, \rho}$. Then,

$\operatorname{Var}\left(h\left(\Lambda_{R}, t ; \varphi_{M, N, \rho}\right)-h\left(\Lambda_{R}, 0 ; \varphi_{M, N, \rho}\right)\right)$

$=\int_{0}^{t} \int_{0}^{t} \operatorname{Cov}\left(\left(N^{+}+N^{-}\right)_{M, N, \rho}\left(\Lambda_{R}, s\right),\left(N^{+}+N^{-}\right)_{M, N, \rho}\left(\Lambda_{R}, s^{\prime}\right)\right) \mathrm{d} s \mathrm{~d} s^{\prime}$

$\leq t^{2} \operatorname{Var}\left(\left(N^{+}+N^{-}\right)_{M, N, \rho}\left(\Lambda_{R}, 0\right)\right) \leq 2 t^{2}\left(\operatorname{Var}\left(N_{M, N, \rho}^{+}\left(\Lambda_{R}, 0\right)\right)+\operatorname{Var}\left(N_{M, N, \rho}^{-}\left(\Lambda_{R}, 0\right)\right)\right)$,

where the two last inequalities hold by Cauchy-Schwarz inequality and by stationarity with respect to time. Therefore, by (3.14) applied for $R=t$,

$$
\limsup _{N \rightarrow \infty} \limsup _{M \rightarrow \infty} \operatorname{Var}\left(h\left(\Lambda_{t}, t ; \varphi_{M, N, \rho}\right)-h\left(\Lambda_{t}, 0 ; \varphi_{M, N, \rho}\right)\right)=\underset{R \rightarrow \infty}{\mathrm{O}}\left(t^{4} \log t\right) .
$$

Now we are going to compare $h\left(\Lambda_{t}, t ; \varphi_{M, N, \rho}\right)-h\left(\Lambda_{t}, 0 ; \varphi_{M, N, \rho}\right)$ with $t^{2} h\left(0,0, t ; \varphi_{M, N, \rho}\right)$, using the logarithmic bound (3.13) on fluctuations. We can write

$$
\begin{gathered}
2 t(2\lfloor t\rfloor+1) h\left(0,0, t ; \varphi_{M, N, \rho}\right)=\sum_{y=-\lfloor t\rfloor}^{\lfloor t\rfloor} \int_{-t}^{t}\left(h\left(0,0, t ; \varphi_{M, N, \rho}\right)-h\left(x, y, t ; \varphi_{M, N, \rho}\right)\right) \mathrm{d} x \\
+\sum_{y=-\lfloor t\rfloor}^{\lfloor t\rfloor} \int_{-t}^{t} \varphi_{M, N, \rho}(x, y) \mathrm{d} x+h\left(\Lambda_{t}, t ; \varphi_{M, N, \rho}\right)-h\left(\Lambda_{t}, 0 ; \varphi_{M, N, \rho}\right) .
\end{gathered}
$$

By Cauchy-Schwarz inequality and by stationarity (3.11), for any $(x, y),\left(x^{\prime}, y^{\prime}\right) \in \Lambda_{t}$,

$$
\begin{aligned}
\limsup _{N \rightarrow \infty} \limsup _{M \rightarrow \infty} \operatorname{Cov}( & \left.h\left(0,0, t ; \varphi_{M, N, \rho}\right)-h\left(x, y, t ; \varphi_{M, N, \rho}\right), h\left(0,0, t ; \varphi_{M, N, \rho}\right)-h\left(x^{\prime}, y^{\prime}, t ; \varphi_{M, N, \rho}\right)\right) \\
\leq & \limsup _{N \rightarrow \infty} \limsup _{M \rightarrow \infty} \sqrt{\operatorname{Var}\left(\varphi_{M, N, \rho}(x, y)\right)} \sqrt{\operatorname{Var}\left(\varphi_{M, N, \rho}\left(x^{\prime}, y^{\prime}\right)\right)} \\
& =\underset{t \rightarrow \infty}{\mathrm{O}}(\log t), \quad \text { by (3.13) }
\end{aligned}
$$

and thus

$$
\limsup _{N \rightarrow \infty} \limsup _{M \rightarrow \infty} \operatorname{Var}\left(\sum_{y=-\lfloor t\rfloor}^{\lfloor t\rfloor} \int_{-t}^{t}\left(h\left(0,0, t ; \varphi_{M, N, \rho}\right)-h\left(x, y, t ; \varphi_{M, N, \rho}\right)\right) \mathrm{d} x\right)=\underset{t \rightarrow \infty}{\mathrm{O}}\left(t^{4} \log t\right) .
$$


By the same argument, we get

$$
\limsup _{N \rightarrow \infty} \limsup _{M \rightarrow \infty} \operatorname{Var}\left(\sum_{y=-\lfloor t\rfloor}^{\lfloor t\rfloor} \int_{-t}^{t} \varphi_{M, N, \rho}(x, y) \mathrm{d} x\right)=\underset{t \rightarrow \infty}{\mathrm{O}}\left(t^{4} \log t\right) .
$$

Therefore, using (6.11), (6.10) and that the variance of the sum of three terms is less than three times the sum of the variances,

$$
(2 t(2\lfloor t\rfloor+1))^{2} \limsup _{N \rightarrow \infty} \limsup _{M \rightarrow \infty} \operatorname{Var}\left(h\left(0,0, t ; \varphi_{M, N, \rho}\right)\right)=\underset{t \rightarrow \infty}{\mathrm{O}}\left(t^{4} \log t\right),
$$

which concludes the proof of the Lemma.

Proof of Lemma 6.5. Since $K$ is compact, for any $\delta>0$, we can cover $K$ by a finite number $l_{\delta} \in \mathbb{N}$ of balls $\mathcal{B}\left(\left(x_{i}, y_{i}\right), \delta\right)_{1 \leq i \leq l_{\delta}}$. Fix $i \in \llbracket 1, l_{\delta} \rrbracket$ and $(x, y) \in \mathcal{B}\left(\left(x_{i}, y_{i}\right), \delta\right)$. For all $Y \in \llbracket\left\lfloor n\left(y_{i}-\delta\right)\right\rfloor,\left\lfloor n\left(y_{i}+\delta\right)\right\rfloor \rrbracket$,

$$
\begin{aligned}
& \left|f_{\rho}(x, y)-\frac{1}{n} \varphi_{M, N, \rho}(n x,\lfloor n y\rfloor)\right| \leq\left|f_{\rho}(x, y)-f_{\rho}\left(x_{i}, y_{i}\right)\right|+\left|f_{\rho}\left(x_{i}, y_{i}\right)-\frac{1}{n} \varphi_{M, N, \rho}\left(n x_{i},\left\lfloor n y_{i}\right\rfloor\right)\right| \\
& +\left|\frac{1}{n} \varphi_{M, N, \rho}\left(n x_{i},\left\lfloor n y_{i}\right\rfloor\right)-\frac{1}{n} \varphi_{M, N, \rho}\left(n x_{i}, Y\right)\right|+\left|\frac{1}{n} \varphi_{M, N, \rho}\left(n x_{i}, Y\right)-\frac{1}{n} \varphi_{M, N, \rho}(n x, Y)\right| \\
& \quad+\left|\frac{1}{n} \varphi_{M, N, \rho}(n x, Y)-\frac{1}{n} \varphi_{M, N, \rho}(n x,\lfloor n y\rfloor)\right| \\
& \leq C_{\rho} \delta+\left|f_{\rho}\left(x_{i}, y_{i}\right)-\frac{1}{n} \varphi_{M, N, \rho}\left(n x_{i},\left\lfloor n y_{i}\right\rfloor\right)\right|+\frac{1}{n}\left|\left(N_{M, N, \rho}^{+}-N_{M, N, \rho}^{-}\right)\left(n I_{x, x_{i}} \times\{Y\}\right)\right| \\
& \leq C_{\rho} \delta+\left|f_{\rho}\left(x_{i}, y_{i}\right)-\frac{1}{n} \varphi_{M, N, \rho}\left(n x_{i},\left\lfloor n y_{i}\right\rfloor\right)\right|+\frac{1}{n}\left(N_{M, N, \rho}^{+}+N_{M, N, \rho}^{-}\right)\left(n I_{x_{i}-\delta, x_{i}+\delta} \times\{Y\}\right),
\end{aligned}
$$

where $C_{\rho}:=\left|\rho_{1}\right|+\left|\rho_{2}\right|+3, N_{M, N, \rho}^{ \pm}(D)$ is the number of antikinks/kinks of $\varphi_{M, N, \rho}$ in a domain $D$ and $I_{a, b}:=[a \wedge b, a \vee b]$ (the second inequality holds because the height slope in the $y$ direction is bounded by 1 ). One could simply choose $Y=\left\lfloor n y_{i}\right\rfloor$ in the last inequality and try to control the variance of $\left(N_{M, N, \rho}^{+}+N_{M, N, \rho}^{-}\right)\left(n I_{x_{i}-\delta, x_{i}+\delta} \times\left\{\left\lfloor n y_{i}\right\rfloor\right\}\right)$ for large $n$ (after sending $M, N$ to infinity) but it is not obvious to get a bound better than $\mathrm{O}(n)$ (which is insufficient). Instead, we average the last inequality for all possible values of $Y$ in $\llbracket\left\lfloor n\left(y_{i}-\delta\right)\right\rfloor,\left\lfloor n\left(y_{i}+\delta\right)\right\rfloor \rrbracket$ in order to get

$$
\begin{aligned}
\sup _{(x, y) \in \mathcal{B}\left(\left(x_{i}, y_{i}\right), \delta\right)} & \left|f_{\rho}(x, y)-\frac{1}{n} \varphi_{M, N, \rho}(n x,\lfloor n y\rfloor)\right| \leq C(\rho) \delta+\left|f_{\rho}\left(x_{i}, y_{i}\right)-\frac{1}{n} \varphi_{M, N, \rho}\left(n x_{i},\left\lfloor n y_{i}\right\rfloor\right)\right| \\
& +\frac{1}{(2 \delta n-1) n}\left(N_{M, N, \rho}^{+}+N_{M, N, \rho}^{-}\right)\left(\left(n x_{i},\left\lfloor n y_{i}\right\rfloor\right)+\Lambda_{n \delta+1}\right),
\end{aligned}
$$

where $\Lambda_{n \delta+1}$ is the rectangle defined as at the beginning of the proof of Lemma 6.4. Now, we know from (3.10), from (3.13) and from Bienaymé-Chebyshev inequality that

$$
\limsup _{N \rightarrow \infty} \limsup _{M \rightarrow \infty} \mathbb{P}\left(\left|f_{\rho}\left(x_{i}, y_{i}\right)-\frac{1}{n} \varphi_{M, N, \rho}\left(n x_{i},\left\lfloor n y_{i}\right\rfloor\right)\right| \geq \varepsilon / 4\right)=\underset{n \rightarrow \infty}{\mathrm{O}}\left(n^{-2} \log n\right) .
$$

Moreover, by (3.14) and by invariance by translation of the stationary measures,

$$
\lim _{N \rightarrow \infty} \lim _{M \rightarrow \infty} \operatorname{Var}\left(\left(N_{M, N, \rho}^{+}+N_{M, N, \rho}^{-}\right)\left(\left(n x_{i},\left\lfloor n y_{i}\right\rfloor\right)+\Lambda_{n \delta+1}\right)\right)=\underset{n \rightarrow \infty}{\mathrm{O}}\left(n^{2} \log n\right) .
$$

Besides, since the sum of the asymptotic kink and antikink densities is equal to the average speed $v(\rho)$,

$$
\lim _{N \rightarrow \infty} \lim _{M \rightarrow \infty} \mathbb{E}\left[\left(N_{M, N, \rho}^{+}+N_{M, N, \rho}^{-}\right)\left(\left(n x_{i},\left\lfloor n y_{i}\right\rfloor\right)+\Lambda_{n \delta+1}\right)\right] \underset{n \rightarrow \infty}{\sim}(2 n \delta)^{2} v(\rho) .
$$


Note that the two previous limits exist as explained in Appendix B. Dividing by $(2 \delta n-1) n$ and using Bienaymé-Chebyshev inequality yields

$$
\begin{array}{r}
\limsup _{N \rightarrow \infty} \limsup _{M \rightarrow \infty} \mathbb{P}\left(\frac { 1 } { ( 2 \delta n - 1 ) n } ( N _ { M , N , \rho } ^ { + } + N _ { M , N , \rho } ^ { - } ) \left(\left(n x_{i},\left\lfloor n y_{i}\right\rfloor\right)\right.\right. \\
\left.\left.+\Lambda_{n \delta+1}\right) \geq 2 \delta v(\rho)+\varepsilon / 4\right) \\
=\underset{n \rightarrow \infty}{\mathrm{O}}\left(n^{-2} \log n\right) .
\end{array}
$$

From (6.12), (6.13) and (6.14), we get that for any $\delta>0$,

$$
\begin{array}{r}
\limsup _{N \rightarrow \infty} \limsup _{M \rightarrow \infty} \mathbb{P}\left(\sup _{(x, y) \in K}\left|f_{\rho}(x, y)-\frac{1}{n} \varphi_{M, N, \rho}(n x,\lfloor n y\rfloor)\right| \geq C_{\rho}^{\prime} \delta+\varepsilon / 2\right) \\
\leq \sum_{i=1}^{l_{\delta}} \limsup _{N \rightarrow \infty} \limsup _{M \rightarrow \infty} \mathbb{P}\left(\sup _{(x, y) \in \mathcal{B}\left(\left(x_{i}, y_{i}\right), \delta\right)}\left|f_{\rho}(x, y)-\frac{1}{n} \varphi_{M, N, \rho}(n x,\lfloor n y\rfloor)\right| \geq C_{\rho}^{\prime} \delta+\varepsilon / 2\right) \\
=\underset{n \rightarrow \infty}{\mathrm{O}}\left(n^{-2} \log n\right),
\end{array}
$$

with $C_{\rho}^{\prime}:=\left|\rho_{1}\right|+\left|\rho_{2}\right|+3+2 v(\rho)$ which concludes the proof by setting $\delta=\varepsilon /\left(2 C_{\rho}^{\prime}\right)$.

\subsection{Conclusion of the proof of Theorem 3.1}

Propositions 5.10 (compactness) and Proposition 6.1 together with Corollary 6.3 provide all necessary ingredients to conclude the proof of Theorem 3.1.

Proposition 6.6. For all $\omega \in \Omega_{0} \cap \Omega_{1}$, all $f \in \bar{\Gamma}$ and all $R, T>0$,

$$
\sup _{|x|,|y| \leq R, t \in[0, T]}\left|S_{n}(0, t ; f, \omega)(x, y)-u(x, y, t)\right| \underset{n \rightarrow \infty}{\longrightarrow} 0,
$$

where $u$ is the unique viscosity solution of (3.5).

Proof. Assume that convergence (6.15) does not hold for some $\omega \in \Omega_{0} \cap \Omega_{1}, f \in \bar{\Gamma}$ and $R, T>0$. Then, there exists $\varepsilon>0$ and a subsequence $\left(n_{k}\right)_{k \in \mathbb{N}}$ such that

$$
\sup _{|x|,|y| \leq R, t \in[0, T]}\left|S_{n_{k}}(0, t ; f, \omega)(x, y)-u(x, y, t)\right| \geq \varepsilon .
$$

By Proposition 5.10, we can extract another subsequence $\left(n_{k_{l}}\right)_{l \in \mathbb{N}}$ such that for all $g \in \bar{\Gamma}$, the sequence $\left(S_{n_{k_{l}}}(\cdot, \cdot ; g, \omega)\right)_{l \in \mathbb{N}}$ converges towards a certain $S(\cdot, \cdot ; g, \omega)$ in $\mathcal{F}_{\mathcal{T}}$. By Proposition 6.1 and Corollary 6.3, $(S(s, t, \cdot ; \omega))_{0 \leq s \leq t \leq T}$ satisfies all sufficient conditions of Proposition 3.5. Therefore, $(x, y, t) \mapsto S(0, t, f ; \omega)(x, y)=u(x, y, t)$ is the unique viscosity solution of (3.3) and thus $(x, y, t) \mapsto S_{n_{k_{l}}}(0, t ; g, \omega)(x, y)$ converges on all compact sets of $\mathbb{R}^{2} \times[0, T]$ towards $u$ when $l$ goes to infinity which is a contradiction with (6.16).

The full proof of Theorem 3.1 follows from Proposition 6.6 and the fact that, by locality (Corollary 4.8),

$$
\sup _{|x|,|y| \leq R, t \in[0, T]}\left|S_{n}(0, t ; f, \omega)(x, y)-\frac{1}{n} h\left(n \cdot,\lfloor n \cdot\rfloor, n t, \varphi_{n} ; \omega\right)\right| \underset{n \rightarrow \infty}{\longrightarrow} 0,
$$

since both rescaled initial height functions $n^{-1} \varphi_{n}^{f}(n \cdot,\lfloor n \cdot\rfloor)$ and $n^{-1} \varphi_{n}(n \cdot,\lfloor n \cdot\rfloor)$ converges to $f$ uniformly on $[-R-\alpha T, R+\alpha T]^{2}$ by (4.15) and assumption (3.3).

\section{A Sufficient conditions for viscosity solutions of Hamilton-Jacobi equations}

In this section, we give a self-contained proof of Proposition 3.5 which is inspired from [22, Lemma 5.3] and [30, Proposition 7.1]. 
Proof. Let us show that $u:(x, t) \mapsto S(0, t, f)(x)$ defined from $\mathbb{R}^{2} \times[0, T]$ to $\mathbb{R}$ is a viscosity solution of (3.5). First of all, by assumption, $u$ in continuous on $\mathbb{R}^{d} \times[0, T]$. Then, by the Semi-group property:

$$
u(\cdot, 0)=S(0,0, g)=g .
$$

We are left to show that $u$ is a subsolution (the proof that $u$ is a supersolution being identical $)$. Let $\phi \in \mathcal{C}^{\infty}\left(\mathbb{R}^{d} \times(0, T)\right)$ and $\left(x_{0}, t_{0}\right) \in \mathbb{R}^{d} \times(0, T)$ such that $\phi\left(x_{0}, t_{0}\right)=u\left(x_{0}, t_{0}\right)$ and $\phi \geq u$ on a neighbourhood of $\left(x_{0}, t_{0}\right)$. At first, we introduce the following affine approximation of $\phi$ around $x_{0}$ :

$$
\psi(x, t):=\phi\left(x_{0}, t\right)+\nabla \phi\left(x_{0}, t_{0}\right) \cdot\left(x-x_{0}\right) .
$$

As $\psi$ and $\phi$ have the same value and derivatives at $\left(x_{0}, t_{0}\right)$, it is enough to show that

$$
\partial_{t} \psi\left(x_{0}, t_{0}\right) \leq v\left(\nabla \psi\left(x_{0}, t_{0}\right)\right)
$$

by studying $\psi\left(x_{0}, t_{0}\right)-\psi\left(x_{0}, t_{0}-\delta\right)$ for small positive $\delta$.

On the one hand, by the semi-group property and the definition of $u$,

$$
\psi\left(x_{0}, t_{0}\right)=u\left(x_{0}, t_{0}\right)=S\left(t_{0}-\delta, t_{0}, u\left(\cdot, t_{0}-\delta\right)\right)\left(x_{0}\right) .
$$

On the other hand, it is easy to show that $\nabla \phi\left(x_{0}, t_{0}\right) \in \mathbb{R} \times[-1,0]$ thanks to the assumptions $\phi \geq u$ around $\left(x_{0}, t_{0}\right)$ with equality at $\left(x_{0}, t_{0}\right)$ and the slopes constraints satisfied by functions in $\bar{\Gamma}$ such as $u\left(\cdot, t_{0}\right)$. Therefore, by compatibility with linear solutions and translation invariance,

$$
S\left(t_{0}-\delta, t_{0}, \psi\left(., t_{0}-\delta\right)\right)\left(x_{0}\right)=\psi\left(x_{0}, t_{0}-\delta\right)+\delta v\left(\nabla \psi\left(x_{0}, t_{0}\right)\right)
$$

We are left to compare $S\left(t_{0}-\delta, t_{0}, \psi\left(., t_{0}-\delta\right)\right)\left(x_{0}\right)$ with $S\left(t_{0}-\delta, t_{0}, u\left(\cdot, t_{0}-\delta\right)\right)\left(x_{0}\right)$. Thanks to locality and monotony, this can be done by comparing $\psi\left(., t_{0}-\delta\right)$ with $u\left(\cdot, t_{0}-\delta\right)$ in the ball $\mathcal{B}\left(x_{0}, \alpha \delta\right)$. By Taylor expansion of $\phi$ and $\psi$ at order 2 around $\left(x_{0}, t_{0}\right)$,

$$
\phi(x, t)=\psi(x, t)+\mathrm{O}\left(\left\|x-x_{0}\right\|_{\infty}^{2}+\left|t-t_{0}\right|^{2}\right) .
$$

Moreover, $u \leq \phi$ on a neighbourhood of $\left(x_{0}, t_{0}\right)$ hence $u\left(\cdot, t_{0}-\delta\right) \leq \phi\left(\cdot, t_{0}-\delta\right)$ on $\mathcal{B}\left(x_{0}, \alpha \delta\right)$ for $\delta$ small enough. Altogether, there exists $C>0$ such that for all $\delta$ small enough,

$$
\forall x \in \mathcal{B}\left(x_{0}, \alpha \delta\right) \quad u\left(x, t_{0}-\delta\right) \leq \psi\left(x, t_{0}-\delta\right)+C \delta^{2} .
$$

Now, we set $g:=u\left(., t_{0}-\delta\right) \wedge \psi\left(., t_{0}-\delta\right)$. By locality property (applied at $x_{0}$ with $R=0)$,

$$
\left|S\left(t_{0}-\delta, t_{0}, u\left(., t_{0}-\delta\right)\right)\left(x_{0}\right)-S\left(t_{0}-\delta, t_{0}, g\right)\left(x_{0}\right)\right| \leq \sup _{x \in \mathcal{B}\left(x_{0}, \alpha \delta\right)}\left|u\left(x, t_{0}-\delta\right)-g(x)\right| \leq C \delta^{2},
$$

where the last inequality holds because of (A.4). Since $\psi \geq g$,

$$
\begin{aligned}
S\left(t_{0}-\delta, t_{0}, \psi\left(., t_{0}-\delta\right)\right)\left(x_{0}\right) & \geq S\left(t_{0}-\delta, t_{0}, g\right)\left(x_{0}\right) \\
& \geq S\left(t_{0}-\delta, t_{0}, u\left(., t_{0}-\delta\right)\right)\left(x_{0}\right)-C \delta^{2} \\
& =\psi\left(x_{0}, t_{0}\right)-C \delta^{2} .
\end{aligned}
$$

Using (A.3), we finally get

$$
\psi\left(x_{0}, t_{0}-\delta\right)+\delta v\left(\nabla \psi\left(x_{0}, t_{0}\right)\right) \geq \psi\left(x_{0}, t_{0}\right)-C \delta^{2}
$$

and then

$$
\partial_{t} \psi\left(x_{0}, t_{0}\right)=\lim _{\delta \rightarrow 0} \frac{\psi\left(x_{0}, t_{0}\right)-\psi\left(x_{0}, t_{0}-\delta\right)}{\delta} \leq v\left(\nabla \psi\left(x_{0}, t_{0}\right)\right) .
$$




\section{B Stationary kink/antikink correlations and proof of (3.14)}

In this section, we give more details about the determinantal structure of the stationary measures introduced in Section 3.3 and show that the kink/antikink correlations are bounded by the inverse of the distance squared in order to deduce (3.14).

Let us first fix $M$ and $N$, the sizes of the torus, and a slope $\rho=\left(\rho_{1}, \rho_{2}\right) \in \mathbb{R} \times(-1,0)$. The existence of a stationary height profile $\varphi_{M, N, \rho}$ (with value fixed e.g. to 0 at the origin) whose average slope approaches $\rho$ was already discussed in Section 3.3. The height function (and in particular the kinks and antikinks) are totally determined by the occupation variables $\eta(x, y)$ for $(x, y) \in \mathbb{R} \times \mathbb{Z}$ that take value 1 if there is a level line of the height function passing by $(x, y)$ (i.e if $\varphi_{M, N, \rho}(x, y+1)-\varphi_{M, N, \rho}(x, y)=-1$ ) and 0 otherwise. In [20], the author showed, that any moments of the occupation variables can be computed thanks to a determinant: for any $\left(x_{1}, y_{1}\right), \cdots,\left(x_{m}, y_{m}\right) \in \mathbb{R} \times \mathbb{Z}$,

$$
\mathbb{E}\left[\eta\left(x_{1}, y_{1}\right) \cdots \eta\left(x_{m}, y_{m}\right)\right]=\operatorname{det}\left(S_{M, N, \rho}\left(x_{k}, y_{k} ; x_{l}, y_{l}\right)\right)_{1 \leq k, l \leq m},
$$

where $S_{M, N, \rho}$ is an explicit kernel that somehow simplifies in the infinite volume limit:

$$
\lim _{N \rightarrow \infty} \lim _{M \rightarrow \infty} S_{M, N, \rho}\left(x^{\prime}, y^{\prime} ; x, y\right)= \begin{cases}\frac{1}{2 \pi} \int_{-\pi \rho_{2}}^{\pi \rho_{2}} e^{\left(x^{\prime}-x\right) \varepsilon(k)} e^{i\left(y^{\prime}-y\right) k} \mathrm{~d} k & \text { for } \quad x^{\prime} \geq x \\ -\frac{1}{2 \pi} \int_{\pi \rho_{2}}^{2 \pi-\pi \rho_{2}} e^{\left(x^{\prime}-x\right) \varepsilon(k)} e^{i\left(y^{\prime}-y\right) k} \mathrm{~d} k & \text { for } \quad x^{\prime}<x,\end{cases}
$$

with $\varepsilon(k)=-\eta_{s} \cos (k)+i \eta_{a} \sin (k)$ and where $\eta_{s}>0, \eta_{a} \in \mathbb{R}$ are parameters uniquely determined by $\rho$. In particular, the law of $\varphi_{M, N, \rho}$ admits an infinite volume limit in the sense that the average of any local function has a limit as $N \rightarrow \infty$ after $M \rightarrow \infty$.

Thanks to this determinental structure, Prähofer and Spohn computed the infinite volume limit of the densities of kinks and antikinks and deduced the speed of growth $v(\rho)$ (defined in (3.6)) depending on the slope $\rho$. Furthemore, they computed the covariance (or "structure function") between kinks, antikinks and occupation variables (see [20, Equation (6.30)] and [19, Equation (27) and (29)]). For our purposes, we only need the antikink/antikink and kink/kink covariances between the origin and $(x, y)$ denoted respectively by $S_{\rho}^{+}(x, y)$ and $S_{\rho}^{-}(x, y)$ and which can be written as:

$$
S_{\rho}^{ \pm}(x, y)=\frac{\eta_{ \pm}^{2}}{(2 \pi)^{2}} \underbrace{\int_{-\rho_{2} \pi}^{\rho_{2} \pi} e^{|x| \varepsilon(k)} e^{i\left(\frac{x}{|x|} y \pm 1\right) k} \mathrm{~d} k}_{A} \times \underbrace{\int_{\rho_{2} \pi}^{2 \pi-\rho_{2} \pi} e^{-|x| \varepsilon\left(k^{\prime}\right)} e^{i\left(\frac{x}{|x|} y \pm 1\right) k^{\prime}} \mathrm{d} k^{\prime}}_{B},
$$

where $\eta_{ \pm}$are positive constants determined by $\rho$. Let us show that

$$
S_{\rho}^{ \pm}(x, y)=\underset{\|(x, y)\| \rightarrow \infty}{\mathrm{O}}\left(\frac{1}{\|(x, y)\|^{2}}\right) .
$$

Without loss of generality, let us treat the case of $S^{+}$and $x \geq 0$. First of all, the modulus of $A$ in (B.3) is bounded by $2 \int_{0}^{\rho_{2} \pi} e^{-\eta_{s} x \cos (k)} \mathrm{d} k$ whose asymptotic behavior for large $x$ only depends on the behavior of the integrand around $\rho_{2} \pi$ where it attains its maximum. Therefore, by a Taylor approximation, we get that for all $(x, y)$,

$$
|A| \leq 2 \int_{0}^{+\infty} e^{-\left(\eta_{s} \cos \left(\rho_{2} \pi\right)-k / C\right) x} \mathrm{~d} k=2 C \frac{e^{-\eta_{s} \cos \left(\rho_{2} \pi\right) x}}{x},
$$

for some constant $C>0$. Now, by integration by parts, we get that

$$
A=\frac{1}{i(y+1)}\left(\left[e^{x \varepsilon(k)} e^{i(y+1) k}\right]_{-\rho_{2} \pi}^{\rho_{2} \pi}-x \int_{-\rho \pi}^{\rho \pi}\left(\eta_{s} \sin (k)+i \eta_{a} \cos (k)\right) e^{x \varepsilon(k)} e^{i(y+1) k} \mathrm{~d} k\right)
$$


and thus, by using (B.5) to bound the second term, we obtain

$$
|A| \leq \frac{1}{|y+1|}\left(2 e^{-\eta_{s} \cos \left(\rho_{2} \pi\right) x}+x \sqrt{\eta_{s}^{2}+\eta_{a}^{2}} 2 C \frac{e^{-\eta_{s} \cos \left(\rho_{2} \pi\right) x}}{x}\right) \leq C^{\prime} \frac{e^{-\eta_{s} \cos \left(\rho_{2} \pi\right) x}}{|y|},
$$

for some constant $C^{\prime}>0$. In any case, we have that

$$
|A|=\underset{\|(x, y)\| \rightarrow \infty}{\mathrm{O}}\left(\frac{e^{-\eta_{s} \cos \left(\rho_{2} \pi\right)|x|}}{\max (|x|,|y|)}\right)
$$

and similar computations show that

$$
|B|=\underset{\|(x, y)\| \rightarrow \infty}{\mathrm{O}}\left(\frac{e^{\eta_{s} \cos \left(\rho_{2} \pi\right)|x|}}{\max (|x|,|y|)}\right),
$$

which concludes the proof of (B.4), by equivalence of norms on $\mathbb{R}^{2}$.

Now, let us show how we can deduce (3.14). The variance of the number of antikinks/kinks in the domain $\Lambda_{R}$ is given by:

$$
\lim _{N \rightarrow \infty} \lim _{M \rightarrow \infty} \operatorname{Var}\left(N_{M, N, \rho}^{ \pm}\left(\Lambda_{R}\right)\right)=\int_{[-R, R]^{2}} \sum_{y, y^{\prime} \in \llbracket-R, R \rrbracket} S_{\rho}^{ \pm}\left(x^{\prime}-x, y^{\prime}-y\right) \mathrm{d} x \mathrm{~d} x^{\prime} .
$$

By standard approximation of sums by integrals arguments and by (B.4), the proof of (3.14) is concluded thanks to the following inequality:

$$
\int_{[-R, R]^{4}} \frac{C_{1}}{\left\|\left(x^{\prime}-x, y^{\prime}-y\right)\right\|^{2}} \vee M \mathrm{~d} x \mathrm{~d} y \mathrm{~d} x^{\prime} \mathrm{d} y^{\prime} \leq \int_{[-R, R]^{2}} C_{2} \log R \mathrm{~d} x \mathrm{~d} y \leq C_{2} R^{2} \log R,
$$

where $M$ is the sup norm of $S_{\rho}^{+}$and $C_{1}, C_{2}>0$ are constants chosen large enough.

\section{Longest light-chain of Poisson points}

In this section we give a control on the maximal length of Poisson points in a domain that can be collected by a light-path (as in Definition 5.1). Let $\omega \in \Omega, k \in \mathbb{N}, \underline{y}=$ $\left(y_{1}, \cdots, y_{k}\right) \in \mathbb{Z}^{k}$ and $D$ a bounded domain of $\mathbb{R}^{2}$. We define the event

$$
C_{\omega, \underline{y}}^{\uparrow}(D):=\left\{\omega \in \Omega, \exists\left(x_{i}, t_{i}\right)_{1 \leq i \leq k} \in \prod_{i=1}^{k}\left(\omega_{y_{i}} \cap D\right), \forall i \in \llbracket 1, k-1 \rrbracket\left|x_{i+1}-x_{i}\right| \leq t_{i+1}-t_{i}\right\},
$$

which means that there exists a light-path that collects at least one point per set $\omega_{y_{i}} \cap D$ in a precise order (from $i=1$ to $i=k$ ). The link with $L^{\uparrow}$ of Definition 5.1 is the following. If $\underline{y}=(y, \cdots, y)$ where $y \in \mathbb{Z}$ appears $k$ times, then

$$
\left\{L^{\uparrow}\left(\omega_{y} \cap D\right) \geq k\right\}=C_{\omega, \underline{y}}^{\uparrow}(D) .
$$

The next Lemma gives a control on the probability of this event when $D$ is a lightrectangle (see Definition 5.1).

Lemma C.1. For any light-rectangle $R \subseteq \mathbb{R}^{2}$, any $k \in \mathbb{N}$ and any $\underline{y}=\left(y_{1}, \cdots, y_{k}\right) \in \mathbb{Z}^{k}$,

$$
\mathbb{P}\left(C_{\omega, \underline{y}}^{\uparrow}(R)\right) \leq\left(\frac{2 e^{2} \operatorname{Leb}(R)}{k^{2}}\right)^{k} .
$$

Proof. This probability is invariant by translation of $R$ and up to a rotation of angle $-\pi / 4$, we can suppose that $R=[0, a] \times[0, b]$ and that where are considering non-decreasing 
paths instead of light-path in the definition of $C^{\uparrow}$. Therefore, by the union bound inequality,

$$
\begin{aligned}
& \mathbb{P}\left(C_{\omega, \underline{y}}^{\uparrow}(R)\right)=\mathbb{P}\left(\exists\left(r_{i}, s_{i}\right)_{1 \leq i \leq k} \in \prod_{i=1}^{k} \omega_{y_{i}}, 0 \leq r_{1} \leq \cdots \leq r_{k} \leq a, 0 \leq s_{1} \leq \cdots \leq s_{k} \leq b\right) \\
& \leq \int_{0 \leq r_{1} \leq \cdots \leq r_{k} \leq a} \int_{0 \leq s_{1} \leq \cdots \leq s_{k} \leq b} \mathbb{P}\left(\bigcap_{i=1}^{k} \#\left\{\omega_{y_{i}} \cap\left[r_{i}, r_{i}+\mathrm{d} r_{i}\right] \times\left[s_{i}, s_{i}+\mathrm{d} s_{i}\right]\right\}=1\right) \\
& \leq \int_{0 \leq r_{1} \leq \cdots \leq r_{k} \leq a} \int_{0 \leq s_{1} \leq \cdots \leq s_{k} \leq b} 2^{k} \mathrm{~d} r_{1} \cdots \mathrm{d} r_{k} \mathrm{~d} s_{1} \cdots \mathrm{d} s_{k}
\end{aligned}
$$

(since the $\omega_{y_{i}}$ are independent PPPs of intensity 2 on $\mathbb{R} \times \mathbb{Z} \times \mathbb{R}_{+}$)

$$
=\frac{(2 a b)^{k}}{(k !)^{2}} \leq\left(\frac{2 e^{2} \operatorname{Leb}(R)}{k^{2}}\right)^{k} \text {. }
$$

In the last inequality, we used that $k ! \geq(k / e)^{k}$ valid for all $k \in \mathbb{N}$ (this classical inequality can be obtained from $e^{x} \geq x^{k} /(k$ !) evaluated at $x=k)$.

Now we give a Corollary that can be useful when dealing with domains different from light-rectangles (the upper bound obtain is not optimal, yet enough for our purposes).

Corollary C.2. For any domain $D \subseteq \mathbb{R}^{2}$, any $k \in \mathbb{N}$ and any $y=\left(y_{0}, \cdots, y_{k}\right) \in \mathbb{Z}^{k+1}$,

$$
\mathbb{P}\left(C_{\omega, \underline{y}}^{\uparrow}(D)\right) \leq 2 \operatorname{Leb}(D)\left(\frac{4 e^{2} \mathrm{v}(D)^{2}}{k^{2}}\right)^{k},
$$

where $\mathrm{v}(D)$ is the vertical diameter of $D$ i.e the longest distance between two points in $D$ aligned vertically.

Proof. In order to realise the event $C_{\omega, y}^{\uparrow}(D)$, once we have chosen $\left(x_{0}, t_{0}\right) \in \omega_{y_{0}} \cap D$, then the rest of the points $\left(x_{1}, t_{1}\right) \cdots\left(x_{k}, t_{k}\right)$ must be in the intersection between $D$ and the cone $\left\{(x, t),\left|x-x_{0}\right| \leq t-t_{0}\right\}$ which is included in a certain light-square denoted $R_{x_{0}, t_{0}}$ whose diagonal is of length less than $2 \mathrm{v}(D)$, hence is of area less than $2 \mathrm{v}(D)^{2}$. By the union bound inequality and Lemma C.1,

$$
\begin{aligned}
\mathbb{P}\left(C_{\omega, \underline{y}}^{\uparrow}(D)\right) & \leq \int_{D} \mathbb{P}\left(C_{\omega,\left(y_{1} \cdots y_{k}\right)}^{\uparrow}\left(R_{x_{0}, t_{0}}\right)\right) 2 \mathrm{~d} x_{0} \mathrm{~d} t_{0} \\
& \leq \int_{D}\left(\frac{2 e^{2} 2 \mathrm{v}(D)^{2}}{k^{2}}\right)^{k} 2 \mathrm{~d} x_{0} \mathrm{~d} t_{0}=2 \operatorname{Leb}(D)\left(\frac{4 e^{2} \mathrm{v}(D)^{2}}{k^{2}}\right)^{k}
\end{aligned}
$$

\section{Compactness for asymptotically continuous functions}

In this section, we show a generalisation of Arzelà-Ascoli theorem, that gives sufficient conditions for "almost continuous functions" (e.g. sequences of functions with jumps of size tending to 0 ) to converge uniformly on all compact sets.

Proposition D.1. Let $\left(f_{n}\right)_{n \in \mathbb{N}}$ be a sequence of functions from a separable metric space $(E, d)$ to a complete metric space $\left(F, d^{\prime}\right)$ such that:

1. Asymptotic equi-continuity: For all $x \in E$ and all $\varepsilon>0$, there exists $\delta>0$ such that

$$
\limsup _{n \rightarrow \infty} \sup _{\substack{y \in E \\ d(x, y) \leq \delta}} d^{\prime}\left(f_{n}(x), f_{n}(y)\right) \leq \varepsilon .
$$

2. Pointwise relative compactness: For all $x \in E$, the sequence $\left(f_{n}(x)\right)_{n \in \mathbb{N}}$ is contained in a compact set of $F$. 
Then, for any subsequence $\left(n_{k}\right)_{k \in \mathbb{N}},\left(f_{n_{k}}\right)_{k \in \mathbb{N}}$ has a subsequence that converges uniformly on all compact subsets of $E$ to a function $f:(E, d) \mapsto\left(F, d^{\prime}\right)$. Moreover, any limit point is continuous.

Proof. For the sake of simplicity and since any subsequence $\left(f_{n_{k}}\right)_{k \in \mathbb{N}}$ still satisfies assumptions 1 and 2, we can assume that $\left(f_{n_{k}}\right)_{k \in \mathbb{N}}=\left(f_{n}\right)_{n \in \mathbb{N}}$ without loss of generality.

Let $E_{0}$ be a dense countable subset of $E$. By pointwise relative compactness and a diagonal extraction argument, we can find a subsequence $\left(n_{l}\right)_{l \in \mathbb{N}}$ such that for every $x \in E_{0},\left(f_{n_{l}}(x)\right)_{l \in \mathbb{N}}$ converges in $F$. Let us show that actually, for all $x \in E,\left(f_{n_{l}}(x)\right)_{l \in \mathbb{N}}$ is a Cauchy sequence, hence converges in $F$. Let $x \in E$ and $\varepsilon>0$. By assumption, there exists $\delta>0$ such that (D.1) is satisfied. By density, we can find $x_{0} \in E_{0}$ such that $d\left(x, x_{0}\right) \leq \delta$. As $\left(f_{n_{l}}\left(x_{0}\right)\right)_{l \in \mathbb{N}}$ converges, it is a Cauchy sequence so for all $l, m$ large enough, $d^{\prime}\left(f_{n_{l}}\left(x_{0}\right), f_{n_{m}}\left(x_{0}\right)\right) \leq \varepsilon$ and thus

$$
\begin{aligned}
d^{\prime}\left(f_{n_{l}}(x), f_{n_{m}}(x)\right) & \leq d^{\prime}\left(f_{n_{l}}(x), f_{n_{l}}\left(x_{0}\right)\right)+d^{\prime}\left(f_{n_{l}}\left(x_{0}\right), f_{n_{m}}\left(x_{0}\right)\right)+d^{\prime}\left(f_{n_{m}}\left(x_{0}\right), f_{n_{m}}(x)\right) \\
& \leq 3 \varepsilon,
\end{aligned}
$$

for $l, m$ large enough by (D.1). Let us call $f$ the pointwise limit. By taking the limit in (D.1), we get immediately that any such limit point is continuous.

Now, let $K$ be a compact subset of $E$ and let us show that $f_{n_{l}}$ converges to $f$ uniformly on $K$. Let $\varepsilon>0$. By compactness and asymptotic equi-continuity assumption, we can find a covering of $K$ by a finite number $p \in \mathbb{N}$ of balls of centers $x_{1}, \cdots x_{p}$ and radius $\delta_{1}, \cdots, \delta_{p}$ such that (D.1) is satisfied with $(x, \delta)=\left(x_{i}, \delta_{i}\right)$ for any $i \in\{1, \cdots p\}$. Therefore, we can find $N \in \mathbb{N}$ such that for all $l \geq N$,

$$
\forall i \in\{1, \cdots, p\} \quad \forall y \in \mathcal{B}\left(x_{i}, \delta\right) \quad d^{\prime}\left(f_{n_{l}}\left(x_{i}\right), f_{n_{l}}(y)\right) \leq \varepsilon .
$$

Moreover, by point-wise convergence we can assume that for all $l \geq N$ and all $i \in$ $\{1, \cdots, p\}, d^{\prime}\left(f_{n_{l}}\left(x_{i}\right), f\left(x_{i}\right)\right) \leq \varepsilon$. Therefore, for all $l \geq N$ and all $y \in K$, if we choose the index $i$ such that $d\left(y, x_{i}\right) \leq \delta_{i}$, then

$$
\begin{aligned}
d^{\prime}\left(f_{n_{l}}(y), f(y)\right) & \leq d^{\prime}\left(f_{n_{l}}(y), f_{n_{l}}\left(x_{i}\right)\right)+d^{\prime}\left(f_{n_{l}}\left(x_{i}\right), f\left(x_{i}\right)\right)+d^{\prime}\left(f\left(x_{i}\right), f(y)\right) \\
& \leq 3 \varepsilon,
\end{aligned}
$$

where we used (D.2) and point-wise convergence in the last inequality.

\section{References}

[1] A-L Barabási and Harry Eugene Stanley. Fractal concepts in surface growth. Cambridge University Press, 1995. MR-1600794

[2] Alexei Borodin and Patrik L Ferrari. Anisotropic KPZ growth in 2+1 dimensions: fluctuations and covariance structure. Journal of Statistical Mechanics, P02009, 2009. MR-2485688

[3] Alexei Borodin and Patrik L Ferrari. Anisotropic growth of random surfaces in 2+1 dimensions. Communications in Mathematical Physics, 325(2):603-684, 2014. MR-3148098

[4] Sunil Chhita and Fabio Lucio Toninelli. A (2+1)-dimensional Anisotropic KPZ growth model with a smooth phase. Communications in Mathematical Physics, 367(2):483-516, 2019. MR-3936124

[5] Ivan Corwin. The Kardar-Parisi-Zhang equation and universality class. Random Matrices: Theory and Applications, 1(01):1130001, 2012. MR-2930377

[6] Ivan Corwin and Fabio Lucio Toninelli. Stationary measure of the driven two-dimensional $q$-Whittaker particle system on the torus. Electron. Commun. Probab., 21:1-12, 2016. MR3510252

[7] Lawrence C Evans. Partial differential equations. American Mathematical Society, 2010. MR-2597943 
[8] Lawrence C Evans. Envelopes and nonconvex Hamilton-Jacobi equations. Calculus of Variations and Partial Differential Equations, 50(1-2):257-282, 2014. MR-3194683

[9] Patrik L Ferrari and Michael Prähofer. One-dimensional stochastic growth and gaussian ensembles of random matrices. In Proceedings of Inhomogeneous Random Systems 2005, Markov Processes and Related Fields, volume 12, pages 203-234, 2006. MR-2249629

[10] Patrik L Ferrari and Herbert Spohn. Random growth models. arXiv preprint arXiv:1003.0881, 2010. MR-2932658

[11] DJ Gates and M Westcott. Stationary states of crystal growth in three dimensions. Journal of Statistical Physics, 81(3-4):681-715, 1995.

[12] John M Hammersley. A few seedlings of research. In Proceedings of the Sixth Berkeley Symposium on Mathematical Statistics and Probability, Volume 1: Theory of Statistics. The Regents of the University of California, 1972. MR-0405665

[13] Hitoshi Ishii. Uniqueness of unbounded viscosity solution of Hamilton-Jacobi equations. Indiana University Mathematics Journal, 33(5):721-748, 1984. MR-0756156

[14] Mehran Kardar, Giorgio Parisi, and Yi-Cheng Zhang. Dynamic scaling of growing interfaces. Physical Review Letters, 56(9):889, 1986.

[15] Richard Kenyon. Lectures on dimers. arXiv preprint arXiv:0910.3129, 2009. MR-2523460

[16] Richard Kenyon, Andrei Okounkov, and Scott Sheffield. Dimers and amoebae. Annals of Mathematics, 1019-1056, 2006. MR-2215138

[17] Martin Legras and Fabio Lucio Toninelli. Hydrodynamic limit and viscosity solutions for a two-dimensional growth process in the anisotropic KPZ class. Communications on Pure and Applied Mathematics, 72(3):620-666, 2018. MR-3911895

[18] Fabio Martinelli. Lectures on Glauber dynamics for discrete spin models. In Lectures on Probability Theory and Statistics, pages 93-191. Springer, 1999. MR-1746301

[19] M Prähofer and H Spohn. An exactly solved model of three-dimensional surface growth in the anisotropic kpz regime. Journal of Statistical Physics, 88(5-6):999-1012, 1997. MR-1478060

[20] Michael Prähofer. Stochastic Surface Growth. PhD thesis, July 2003.

[21] Jeremy Quastel. Introduction to KPZ. Current Developments in Mathematics, 2011(1), 2011. MR-3098078

[22] Fraydoun Rezakhanlou. Continuum limit for some growth models II. Annals of Probability, 1329-1372, 2001. MR-1872745

[23] Fraydoun Rezakhanlou. Continuum limit for some growth models. Stochastic Processes and Their Applications, 101(1):1-41, 2002. MR-1921440

[24] Timo Seppäläinen. A microscopic model for the Burgers equation and longest increasing subsequences. Electronic Journal of Probability, 1, 1996. MR-1386297

[25] Timo Seppäläinen. Strong law of large numbers for the interface in ballistic deposition. In Annales de l'Institut Henri Poincare (B) Probability and Statistics, volume 36(6), pages 691-736. Elsevier, 2000. MR-1797390

[26] Timo Seppäläinen. Translation Invariant Exclusion Processes (Book in Progress). http://www. math.wisc.edu/ seppalai/excl-book/etusivu.html, 2008.

[27] Fabio Lucio Toninelli. A (2+1)-dimensional growth process with explicit stationary measures. The Annals of Probability, 45(5):2899-2940, 2017. MR-3706735

[28] Fabio Lucio Toninelli. (2+1)-dimensional interface dynamics: mixing time, hydrodynamic limit and Anisotropic KPZ growth. Proceedings of the International Congress of Mathematicians 2018, Rio de Janeiro, 2:2719-2744, 2018. MR-3966870

[29] Dietrich E Wolf. Kinetic roughening of vicinal surfaces. Physical Review Letters, 67(13):1783, 1991.

[30] Xufan Zhang. Domino shuffling height process and its hydrodynamic limit. arXiv preprint arXiv:1808.07409, 2018.

Acknowledgments. I would like to thank my advisor Fabio Toninelli for introducing me to the domain of hydrodynamic limits, for frequent discussions and for very careful proofreading. I also want to thank Xufan Zhang for explaining details about his article [30] and the anonymous referee for a careful reading and for constructive comments. 


\section{Electronic Journal of Probability Electronic Communications in Probability}

\section{Advantages of publishing in EJP-ECP}

- Very high standards

- Free for authors, free for readers

- Quick publication (no backlog)

- Secure publication $\left(\mathrm{LOCKSS}^{1}\right)$

- Easy interface (EJMS²)

\section{Economical model of EJP-ECP}

- Non profit, sponsored by $\mathrm{IMS}^{3}, \mathrm{BS}^{4}$, ProjectEuclid ${ }^{5}$

- Purely electronic

\section{Help keep the journal free and vigorous}

- Donate to the IMS open access fund ${ }^{6}$ (click here to donate!)

- Submit your best articles to EJP-ECP

- Choose EJP-ECP over for-profit journals

\footnotetext{
${ }^{1}$ LOCKSS: Lots of Copies Keep Stuff Safe http://www. lockss.org/

${ }^{2}$ EJMS: Electronic Journal Management System http://www.vtex.lt/en/ejms.html

${ }^{3}$ IMS: Institute of Mathematical Statistics http://www.imstat.org/

${ }^{4}$ BS: Bernoulli Society http://www. bernoulli-society.org/

${ }^{5}$ Project Euclid: https://projecteuclid.org/

${ }^{6}$ IMS Open Access Fund: http://www.imstat.org/publications/open.htm
} 